Cahiers
de la Recherche
surles Droits Fondamentaux

\section{Cahiers de la recherche sur les droits} fondamentaux

15 | 2017

Le corps humain saisi par le droit : entre liberté et propriété

\title{
Chronique de jurisprudence constitutionnelle
}

française 2016

A Chronicle of French Constitutional Case Law 2016

Aurore Catherine, Anne-Sophie Denolle et Eugénie Duval

\section{(2) OpenEdition}

\section{Journals}

Édition électronique

URL : https://journals.openedition.org/crdf/569

DOI : $10.4000 /$ crdf.569

ISSN : 2264-1246

Éditeur

Presses universitaires de Caen

Édition imprimée

Date de publication : 1 novembre 2017

Pagination : 155-175

ISBN : 978-2-84133-858-0

ISSN : 1634-8842

Référence électronique

Aurore Catherine, Anne-Sophie Denolle et Eugénie Duval, « Chronique de jurisprudence

constitutionnelle française $2016 »$, Cahiers de la recherche sur les droits fondamentaux [En ligne], 15 |

2017, mis en ligne le 01 octobre 2019, consulté le 16 novembre 2022. URL : http://

journals.openedition.org/crdf/569; DOI : https://doi.org/10.4000/crdf.569 


\title{
Chronique de jurisprudence constitutionnelle française 2016
}

\author{
Aurore CATHERINE \\ Maître de conférences en droit public à l'université de Caen Normandie \\ Centre de recherche sur les droits fondamentaux et les évolutions du droit (CRDFED, EA 2132)
}

\section{Anne-Sophie DENOLLE}

Maître de conférences en droit public à l'université de Nîmes

Détection, évaluation et gestion des risques CHROniques et éMErgents (CHROME, EA 7352)

\section{Eugénie DUVAL}

Doctorante en droit public à l'université de Caen Normandie

Centre de recherche sur les droits fondamentaux et les évolutions du droit (CRDFED, EA 2132)

I. Une jurisprudence globalement protectrice des droits et libertés civils et politiques

A. Un état d'urgence "permanent " à l'épreuve du contrôle de proportionnalité du juge constitutionnel

B. Droit pénal: une protection accrue des droits et libertés dans le cadre de la procédure pénale mais une jurisprudence peu lisible dans le domaine économique et financier

C. Le renforcement des droits des collectivités

II. La protection par le Conseil des droits économiques et sociaux: l'art de ménager la chèvre et le chou

A. Les droits des travailleurs: une jurisprudence en demi-teinte

B. Les avancées en demi-teinte en matière de logement et de santé

La session jurisprudentielle 2016 du Conseil constitutionnel a été marquée, comme chaque année, par une diversité de décisions relayant des questions d'ordre substantiel et procédural. Ce fut également l'occasion pour le Conseil d'adopter un nouveau mode de rédaction de ses décisions et de rappeler certains éléments quant aux conditions de sa saisine dans le cadre d'une question prioritaire de constitutionnalité (QPC) ${ }^{1}$. Cette chronique n'a pas vocation à être exhaustive et cherche davantage à livrer au lecteur une analyse contextualisée des décisions qui ont retenu l'attention des auteurs.

Les droits civils et politiques ont fait, comme à l'accoutumée, l'objet d'une jurisprudence abondante, qui s'est avérée globalement protectrice, bien qu'elle ne soit pas exempte de certaines critiques (I). Le Conseil a naturellement été amené, dans un contexte d'état d'urgence devenu

1. Voir notamment CC, déc. n 2015-513/514/526 QPC du 14 janvier 2016, M. Alain D. et autres. 
permanent, à se prononcer à plusieurs reprises sur ce sujet. Si la permanence actuelle de cette situation exceptionnelle fait l'objet de très nombreuses critiques, il n'en demeure pas moins que le Conseil est très vigilant dans le domaine en exerçant un contrôle de proportionnalité des dispositions en vue d'assurer une protection effective des droits et libertés susceptibles d'être menacés (A). L'année 2016 se caractérise encore par une jurisprudence nourrie en matière pénale. S’agissant de la procédure pénale, les décisions rendues apparaissent comme étant plutôt favorables aux droits des personnes. La rencontre entre le droit pénal et le domaine économique et financier semble toutefois plus problématique, le Conseil semblant à cet égard avancer à tâtons, rendant ainsi sa jurisprudence peu lisible (B). En revanche, pour ce qui concerne les collectivités locales, le contrôle du Conseil permet de préciser, voire parfois de renforcer, les droits qui leur sont attachés, et notamment celui de leur libre administration (C).

Il nous a semblé par ailleurs que le Conseil avait réservé une jurisprudence de premier choix aux droits économiques et sociaux protégés par la Constitution, constat qui, à l'issue de notre analyse, est finalement apparu beaucoup moins évident (II). Alors qu'ils n'occupent traditionnellement pas le devant de la scène en droit constitutionnel, les droits des travailleurs, notamment ceux protégés par le préambule de la Constitution de 1946, ont fait l'objet de nombreuses décisions en 2016 dont certaines méritent d'être particulièrement mises en exergue (A). Par ailleurs, les domaines du logement et de la santé ont été l'occasion pour le Conseil de valider des réformes majeures sans pour autant être à l'abri de certaines déclarations d'inconstitutionnalité ou de réserves d'interprétation (B).

\section{Une jurisprudence globalement protectrice des droits et libertés civils et politiques}

\section{A. Un état d'urgence "permanent" à l'épreuve du contrôle de proportionnalité du juge constitutionnel}

L'état d'urgence, dont le régime est issu de la loi no 55-385 du 3 avril 1955, a été réformé à plusieurs reprises suite aux attentats survenus en France le 13 novembre 2015. La conséquence en a été l'augmentation des pouvoirs de police des autorités administratives: en volumétrie, d'abord, car les mesures de police administrative prises dans le cadre de l'état d'urgence pour sauvegarder l'ordre public se sont multipliées; sur le fond ensuite, car la loi a conféré des pouvoirs supplémentaires aux autorités administratives et au juge administratif.
C'est dans ce cadre que le Conseil constitutionnel a été saisi à plusieurs reprises en 2016 par le juge administratif sur le fondement de l'article 61-1 de la Constitution, permettant au juge constitutionnel d'opérer un contrôle a posteriori des lois relatives à l'état d'urgence. En effet, le Conseil constitutionnel n'a été saisi de la question de la constitutionnalité des lois relatives à l'état d'urgence que dans le cadre de QPC, aucune saisine a priori n'ayant eu lieu. Toutes ces décisions permettent de conforter le régime légal de l'état d'urgence et d'en assurer les exigences constitutionnelles.

Dans la continuité des décisions qu'il a rendues en la matière en 2015, le Conseil constitutionnel s'est tout d'abord prononcé dans deux décisions du 19 février 2016. Dans la première, il s'est prononcé sur l'interdiction de réunion et de manifestation et, dans la seconde, sur les perquisitions. Sont à noter plusieurs points communs : la confirmation du caractère de mesures de police administrative des mesures de fermeture provisoire des salles de spectacles, débits de boissons et lieux de réunion ou d'interdiction de réunion et des perquisitions; l'affirmation de la compétence du juge administratif pour contrôler les mesures prises et la conciliation entre plusieurs droits et libertés.

Dans la décision nº 2016-535 QPC du 19 février 2016², le Conseil commence par rappeler au considérant 3 que «la Constitution n'exclut pas la possibilité pour le législateur de prévoir un régime d'état d'urgence ${ }^{3}$, écartant ainsi la nécessité de constitutionnaliser l'état d'urgence comme cela fit l'objet d'un projet de réforme. Pour couper court à toute polémique sur la pérennisation de l'état d'urgence (l'un des arguments pour en assurer la constitutionnalisation), le Conseil en profite pour affirmer que la loi «ne saurait être excessive au regard du péril imminent ou de la calamité publique ayant conduit à la déclaration de l'état d'urgence ${ }^{4}$. Il envisage ainsi pouvoir exercer, également dans le cadre de son contrôle a priori si l'occasion se présentait, un contrôle de proportionnalité d'une telle loi au regard de cet objectif.

Le Conseil admet ensuite que le pouvoir de l'autorité administrative d'ordonner, dans le cadre de l'état d'urgence, la fermeture provisoire de lieux de réunion et d'interdire les réunions de nature à provoquer ou à entretenir le désordre, en ce qu'il restreint la liberté de se réunir, peut porter «atteinte au droit d'expression collective des idées et des opinions » garanti par l'article 11 de la Déclaration des droits de l'homme et du citoyen $(\mathrm{DDHC})^{5}$. Une telle atteinte est jugée acceptable à la condition que les mesures dont il est question, qui présentent un caractère individuel, soient motivées et que le juge administratif s'assure que «chacune de ces mesures est adaptée, nécessaire et proportionnée à la finalité qu'elle

2. CC, déc. $\mathrm{n}^{\circ} \mathbf{2 0 1 6 - 5 3 5}$ QPC du 19 février 2016, Ligue des droits de l'homme.

3. Déjà affirmée dans la décision $\mathrm{n}^{\circ} 85-187 \mathrm{DC}$ du 25 janvier 1985, Loi relative à l'état d'urgence en Nouvelle-Calédonie, cons. 4 ; rappelée dans la décision n 2015-527 QPC du 22 décembre 2015, M. Cédric D., cons. 8 et la décision nº 2016-536 QPC du 19 février 2016, Ligue des droits de l'homme, cons. 5.

4. CC, déc. $\mathrm{n}^{\circ}$ 2016-535 QPC, cons. 9.

5. Ibid., cons. 6 . 
poursuit $»^{6}$. Le Conseil précise en ce sens qu'un recours peut être formé à l'encontre de la décision du ministre de l'Intérieur ou du préfet, "y compris par la voie du référé » ${ }^{7}$. Il en conclut que

[...] les dispositions contestées, qui ne sont pas entachées d'incompétence négative, opèrent une conciliation qui n'est pas manifestement déséquilibrée entre le droit d'expression collective des idées et des opinions et l'objectif de valeur constitutionnelle de sauvegarde de l'ordre public ${ }^{8}$.

La professeure Hélène Surrel rapproche cette jurisprudence de celle de la Cour européenne des droits de l'homme ${ }^{9}$ qui est particulièrement attentive au respect de certaines garanties, notamment l'exigence de motivation des décisions et l'existence d'un contrôle juridictionnel pour éviter d'éventuels abus ${ }^{10}$. Il faut également mettre en exergue qu'en procédant de la sorte, le juge constitutionnel procède par interprétations directives: il précise en effet les garanties nécessaires à la sauvegarde du droit considéré dont le juge administratif devra veiller au respect ${ }^{11}$.

Dans la décision no 2016-536 QPC du 19 février 2016 relative aux perquisitions et saisies administratives dans le cadre de l'état d'urgence, était contesté le paragraphe I de l'article 11 de la loi du 3 avril 1955 dans sa rédaction résultant de la loi du 20 novembre 2015. Celui-ci serait, selon les requérants, contraire à la liberté individuelle, au droit au respect de la vie privée et au droit à un recours juridictionnel effectif. Sont par ailleurs dénoncées l'incompétence négative du législateur et la violation du principe de séparation des pouvoirs. Le Conseil constitutionnel déclare constitutionnelles les dispositions relatives aux perquisitions. Il juge notamment leur conformité à l'article 66 de la Constitution: il reprend en ce sens les termes de sa décision $n^{\circ}$ 2015-527 QPC. La nature préventive de ces mesures lui permet d'affirmer que «ces perquisitions administratives n'ont pas à être placées sous la direction et le contrôle de l'autorité judiciaire $»^{12}$. De même, il considère que la disposition contestée ne porte pas atteinte au droit à l'inviolabilité du domicile et est conforme aux articles 2 et 16 de la DDHC. Si une atteinte est portée à ce droit, elle est admissible dans la mesure où elle est conditionnée et assortie de garanties ${ }^{13}$. Celles-ci permettent de déclarer la constitutionnalité des dispositions en cause. L'interprétation constructive de la loi par le juge constitutionnel ${ }^{14}$ permet ainsi de considérer que la disposition litigieuse ne porte pas d'atteintes disproportionnées aux droits à l'inviolabilité du domicile et à un recours juridictionnel effectif. Tout d'abord, le champ d'application des perquisitions est limité aux lieux situés dans la zone couverte par l'état d'urgence. Ensuite, la décision doit préciser le lieu et le moment de la perquisition qui doit être conduite en présence d'un officier de police judiciaire et de l'occupant (ou à défaut de son représentant ou de deux témoins). La perquisition doit donner lieu à un compte rendu qui doit être communiqué sans délai au procureur de la République. Le Conseil insiste tout particulièrement sur le fait que la décision qui ordonne une perquisition ainsi que les conditions de sa mise en œuvre doivent être justifiées et proportionnées par rapport aux raisons qui l'ont motivée, et cela surtout pour une perquisition de nuit. Celle-ci doit être fondée sur l'urgence ou l'impossibilité de l'effectuer le jour. Le juge administratif devra ainsi opérer un contrôle minutieux de l'ensemble de ces éléments pour juger de la légalité de la mesure mise en œuvre.

Parallèlement à ce raisonnement, certains éléments de la disposition sont déclarés non conformes à la Constitution. Est ainsi déclarée inconstitutionnelle la disposition permettant à l'autorité administrative de copier les données informatiques auxquelles il aura été possible d'accéder au cours de cette perquisition. Précisément, le Conseil constitutionnel la juge contraire à l'article $2 \mathrm{de}$ la DDHC. Le fait de copier les données informatiques mais sans saisir à proprement parler les ordinateurs a été considéré comme «assimilable à une saisie » ${ }^{15}$. Le Conseil considère que le législateur n'avait pas prévu de dispositions permettant d'assurer une conciliation équilibrée entre l'objectif de valeur constitutionnelle de sauvegarde de l'ordre public et le droit au respect de la vie privée. Là encore, le juge constitutionnel se fait l' «écho $~^{16}$ de la Cour européenne des droits de l'homme qui vérifie l'existence de "garanties adéquates et suffisantes contre les abus».

Le Conseil constitutionnel a eu encore à préciser ces divers éléments dans le cadre d'une troisième QPC lors du dernier trimestre de l'année 2016. Dans la décision $\mathrm{n}^{\circ}$ 2016-567/568 QPC du 23 septembre 2016, il considère que le législateur, « en ne soumettant le recours aux perquisitions à aucune condition et en n'encadrant leur mise en œuvre d'aucune garantie», n'a manifestement pas assuré « une conciliation équilibrée entre l'objectif de valeur constitutionnelle de sauvegarde de l'ordre public et le droit au respect de la vie privée » en particulier de l'inviolabilité du domicile ${ }^{17}$. Il conclut à l'inconstitutionnalité du $1^{\circ} \mathrm{de}$

6. Ibid., cons. 8 .

7. Ibid., cons. 14

8. Ibid., cons. 10

9. H. Surrel, «Chronique de jurisprudence constitutionnelle», Les nouveaux cahiers du Conseil constitutionnel, $\mathrm{n}^{\circ}$ 52, juin 2016, p. 162-163.

10. Cour EDH, 12 juillet 2005, Güneri et autres c. Turquie, $\mathrm{n}^{\circ}$ 45454/99, $\$ 77$ et 79 .

11. Voir en ce sens A. Roux, «État d'urgence et Constitution», in P. Monge, S. Lamouroux, X. Philippe, K. Picard, A. Roux, J.-B. Perrier, N. Catelan, "Jurisprudence du Conseil constitutionnel», Revue française de droit constitutionnel, $\mathrm{n}^{\circ}$ 107, 2016, p. 693.

12. CC, déc. $\mathrm{n}^{\circ}$ 2016-536 QPC, cons. 4 .

13. Ibid., cons. 8 à 11 .

14. Voir A. Roux, «État d'urgence et Constitution», p. 697.

15. CC, déc. no 2016-536 QPC, cons. 14 .

16. H. Surrel, «Chronique de jurisprudence constitutionnelle», p. 159.

17. CC, déc. no 2016-567/568 QPC du 23 septembre 2016, M. Georges F. et autre, cons. 8. 
l'article 11 de la loi du 3 avril 1955 relative à l'état d'urgence dans sa rédaction résultant de l'ordonnance du 15 avril 1960 et se conforme ainsi à la jurisprudence européenne qui exige des "garanties adéquates et suffisantes contre l'arbitraire», surtout lorsque les autorités sont habilitées à effectuer des perquisitions sans mandat judiciaire, ce qui implique alors « un encadrement légal et une limitation des plus stricts de tels pouvoirs ${ }^{18}$. Cette disposition est contraire à l'article 2 de la DDHC de 1789. En effet, le législateur n'a assorti le recours aux perquisitions d'aucune condition et n'a encadré leur mise en œuvre d'aucune garantie. Il n'a ainsi pas permis d'assurer une conciliation équilibrée entre l'objectif de valeur constitutionnelle de sauvegarde de l'ordre public et le droit au respect de la vie privée.

Enfin, dans sa décision $\mathrm{n}^{\circ}$ 2016-600 QPC du 2 décembre 2016, le Conseil procède à la censure partielle de l'article 11 de la loi du 3 avril 1955 relatif à la possibilité de procéder à des perquisitions en période d'état d'urgence. Étaient contestées les modalités de ces perquisitions administratives (saisies, exploitations, conservation de données informatiques) au regard des droits de propriété et du respect de la vie privée. Le Conseil considère qu'il n'y a pas de conciliation manifestement déséquilibrée entre le droit au respect de la vie privée ou le droit de propriété et l'objectif de valeur constitutionnelle de sauvegarde de l'ordre public. Précisément, le Conseil constitutionnel a jugé que le législateur a, en assortissant la saisie et l'exploitation de données informatiques d'un certain nombre de garanties, assuré une conciliation qui n'est pas manifestement déséquilibrée entre le droit au respect de la vie privée et l'objectif de valeur constitutionnelle de sauvegarde de l'ordre public. Il a également jugé que le législateur n'avait pas méconnu le droit à un recours juridictionnel effectif. Concernant la conservation des données informatiques, le Conseil a relevé que le législateur avait encadré les conditions de conservation des données autres que celles caractérisant la menace ayant justifié la saisie en prévoyant un délai à l'issu duquel elles sont détruites. De même, lorsque l'exploitation des données conduit à la constatation d'une infraction, la loi prévoit qu'elles sont conservées selon les règles applicables en matière de procédure pénale. Le Conseil a, en revanche, constaté que lorsque les données copiées caractérisent une menace sans conduire à la constatation d'une infraction, le législateur n'a prévu aucun délai, après la fin de l'état d'urgence, à l'issue duquel ces données sont détruites. Le Conseil a en conséquence jugé que le législateur n'a, en ce qui concerne la conservation de ces données, pas prévu de garanties légales propres à assurer une conciliation équilibrée entre le droit au respect de la vie privée et l'objectif de valeur constitutionnelle de sauvegarde de l'ordre public. Le Conseil a ainsi déclaré contraires à la Constitution les mots: «À l'exception de celles qui caractérisent la menace que constitue pour la sécurité et l'ordre publics le comportement de la personne concernée» figurant à la dernière phrase du huitième alinéa du paragraphe I de l'article 11 de la loi du 3 avril $1955^{19}$. Il a toutefois reporté les effets de cette déclaration d'inconstitutionnalité au $1^{\text {er }}$ mars 2017.

\section{B. Droit pénal: une protection accrue des droits et libertés dans le cadre de la procédure pénale mais une jurisprudence peu lisible dans le domaine économique et financier}

La jurisprudence constitutionnelle en matière pénale a été riche en 2016, apparaissant comme étant plutôt protectrice des droits et libertés.

Parmi les décisions rendues, deux sont relatives à des mesures d'incarcération. Le Conseil a appliqué le principe fondamental reconnu par les lois de la République (PFRLR) de justice pénale des mineurs pour censurer une disposition de l'ordonnance $n^{\circ}$ 454-174 du 2 février 1945 permettant au juge des enfants et au tribunal pour enfants d'ordonner l'exécution provisoire de leurs décisions, même en cas d'opposition ou d'appel. Si le Conseil trouve justifiée "l'exécution provisoire des mesures ou sanctions éducatives et des peines ${ }^{20}$ qui vise à contribuer «à l'objectif de leur [les mineurs] relèvement éducatif et moral ${ }^{21}$, il déclare toutefois contraire à la Constitution la possibilité de prononcer «l'exécution provisoire d'une peine d'emprisonnement sans sursis prononcée à l'encontre d'un mineur, alors que celui-ci comparaît libre devant le tribunal pour enfants $»^{22}$.

Dans une autre décision, le Conseil a précisé ${ }^{23}$, à l'occasion de plusieurs réserves d'interprétation, le dispositif prévu pour les personnes incarcérées à l'occasion d'une demande d'extradition. Il relève que le magistrat du siège, qui peut dans ce cadre prononcer l'incarcération de la personne ou la laisser en liberté en la soumettant à des mesures de contrôle, peut également laisser la personne en liberté «sans mesure de contrôle dès lors que celle-ci présente des garanties suffisantes de représentation ${ }^{24}$. Cette réserve s'avère "conforme à l'intention du législateur $»^{25}$. Les membres du Conseil ont également affirmé que

18. Cour EDH, 10 novembre 2015 , Slavov et autres c. Bulgarie, $\mathrm{n}^{\circ} 58500 / 10, \$ 144$.

19. CC, déc. $\mathrm{n}^{\circ}$ 2016-60o QPC du 2 décembre 2016, M. Raïme A., $\$ 16$.

20. CC, déc. $\mathrm{n}^{\circ}$ 2016-601 QPC du 9 décembre 2016, M. Ibrahim B., $\$ 7$.

21. Ibid.

22. Ibid., $\$ 8$.

23. Voir D. Brach-Thiel, «Utiles précisions autour du placement sous écrou extraditionnel», L'actualité juridique. Droit pénal, 2016, p. 596-597.

24. CC, déc. n 2016-561/562 QPC du 9 septembre 2016, M. Mukhtar A., \$12.

25. Commentaire de la décision $n^{\circ} 561 / 562$ QPC, p. 13, en ligne: http://www.conseil-constitutionnel.fr/conseil-constitutionnel/root/bank/download/ 2016561_562QPC2016561_562qpc_ccc.pdf. 
[...] le respect des droits de la défense exige que la personne présentée au premier président de la cour d'appel ou au magistrat qu'il a désigné puisse être assistée par un avocat et avoir, le cas échéant, connaissance des réquisitions du procureur général ${ }^{26}$.

Enfin, alors même que le législateur n'a pas prévu «de durée maximum au placement sous écrou extraditionnel» ni «d'obligation d'un réexamen périodique du bien-fondé de la détention par un juge ${ }^{27}$, la personne peut «solliciter à tout instant de la procédure [...] sa mise en liberté devant la chambre de l'instruction ${ }^{28}$. Le Conseil indique alors qu'à cette occasion le juge doit contrôler la durée de l'incarcération de la personne, «en tenant compte notamment des éventuels recours exercés par la personne et des délais dans lesquels les autorités juridictionnelles et administratives ont statué $»^{29}$. En outre, si «la durée totale de la détention, dans le cadre de la procédure d'extradition, excède un délai raisonnable», le Conseil relève que l'autorité judiciaire doit alors faire droit à la demande de mise en liberté ${ }^{\circ}$. Le dispositif d'écrou extraditionnel se voit ainsi enrichi de précisions importantes, renforçant les droits des personnes dont l'extradition est demandée.

Le juge de la loi a également, selon une jurisprudence constante, censuré plusieurs dispositions du Code de procédure pénale pour méconnaissance du droit au recours ou encore des droits de la défense et du principe du contradictoire. Parmi ces décisions, la QPC posée par l'association Observatoire international des prisons a permis de consolider les droits des personnes incarcérées pendant la détention provisoire, plus particulièrement leur droit au recours en cas de refus de permis de visite et d'autorisation de téléphoner ${ }^{31}$. Le Conseil considère qu'en dehors de l'hypothèse d'une décision relative au refus d'accorder «un permis de visite au profit d'un membre de la famille du prévenu " ${ }^{32}$, «l'absence de voie de droit permettant la remise en cause de la décision du magistrat [...] conduit à ce que la procédure contestée méconnaisse les exigences découlant de l'article 16 de la Déclaration de 1789", plus particulièrement le droit au recours mais également le droit au respect de la vie privée et celui de mener une vie familiale normale ${ }^{33}$. Cette décision s'inscrit ainsi dans la jurisprudence constante du Conseil s'agissant du droit au recours, selon laquelle «c'est l'absence de recours qui est potentiellement contraire à la Constitution, plutôt que l'existence de règles encadrant ce recours ${ }^{34}$. Le Conseil constitutionnel précise également que l'absence de délai imparti au juge d'instruction pour répondre à une demande de permis de visite effectuée par un membre de la famille de la personne placée en détention provisoire conduit «à priver la personne ayant formé une demande de permis de visite de toute voie de recours dans l'attente d'une réponse à sa demande $»^{35}$.

Des dispositions du Code de procédure pénale ayant «pour effet de priver les parties non assistées par un avocat de la possibilité d'avoir connaissance des réquisitions du ministère public devant la chambre de l'instruction $»^{36}$ ont également été censurées. Conformément à sa jurisprudence antérieure ${ }^{37}$, le Conseil considère que ces dispositions instaurent une différence de traitement entre les parties assistées d'un avocat et celles qui ne le sont pas. Or, cette différence de traitement n'est selon lui pas justifiée et méconnaît ainsi le principe du contradictoire et les droits de la défense ${ }^{38}$.

Dans une décision rendue le 23 septembre 2016, les juges de la rue de Montpensier se sont prononcés en faveur des droits des personnes pouvant faire l'objet d'une transaction pénale, bien que «certaines zones d'ombre subsistent ${ }^{39}$. Le Conseil a notamment censuré pour incompétence négative dans cette décision la disposition qui renvoyait au pouvoir réglementaire le soin de délimiter le champ d'application de la procédure de transaction pénale ${ }^{40}$. Surtout, il a indiqué que la personne suspectée d'avoir commis une infraction doit avoir été informée de son droit à être assistée par un avocat avant d'accepter une proposition de transaction, "y compris si celle-ci intervient pendant qu'elle est placée en garde à vue» ${ }^{41}$. Le Conseil «a

26. CC, déc. n²016-561/562 QPC, $\$ 13$.

27. Ibid, $\$ 19$.

28. Ibid., $\$ 20$.

29. Ibid., $\$ 21$.

30. Ibid.

31. Voir N. Ferran, C. Redon, «Refus de permis de visite et d'autorisation de téléphoner: vers la fin d'un déni de justice», Dedans-dehors, n 92 , juillet 2016, p. 44-45

32. CC, déc. $\mathrm{n}^{\circ}$ 2016-543 QPC du 24 mai 2016, Section française de l’observatoire international des prisons, \$14.

33. Ibid.; aucune voie de recours n'est prévue s'agissant des décisions refusant un permis de visite «demandé au cours de l'instruction par une personne qui n'est pas membre de la famille [...] en l'absence d'instruction ou après la clôture de celle-ci»; il en est de même pour les « décisions refusant l'accès au téléphone à une personne placée en détention provisoire» (ibid., $\$ 12$ et 13).

34. Commentaire de la décision $n^{\circ}$ 2016-543 QPC, p. 11, en ligne: http://www.conseil-constitutionnel.fr/conseil-constitutionnel/root/bank/ download/2016543QPC2016543qpc_ccc.pdf.

35. Ibid., p. 16.

36. CC, déc. $n^{\circ}$ 2016-566 QPC du 16 septembre 2016, $M^{\text {me }}$ Marie-Lou B. et autre, $\$ 9$.

37. C'est «la troisième fois depuis l'existence de la question prioritaire de constitutionnalité [que] le Conseil constitutionnel censure une disposition procédurale qui conditionne la communication de pièces à l'assistance obligatoire de l'avocat» (A.-S. Chavent-Leclère, «Inconstitutionnalité de l'assistance obligatoire de l'avocat », Procédures, n 11, novembre 2016, commentaire 338).

38. CC, déc. no 2016-566 QPC.

39. Voir J.-B. Perrier, «La transaction pénale et les progrès du Conseil constitutionnel», Recueil Dalloz, 2016, p. 2545 sq.

40. CC, déc. $\mathrm{n}^{\circ}$ 2016-569 QPC du 23 septembre 2016, Syndicat de la magistrature et autre, $\$ 17$.

41. Ibid., $\$ 9$ 
ainsi précisé sa jurisprudence en matière de transaction pénale ${ }^{42}$. Les droits de la défense s'appliquent en effet à cette procédure de transaction qui, même si elle «n'aboutit pas à une sanction ayant le caractère d'une punition, [...] n'est pas étrangère au domaine répressif, puisqu'elle a pour objet l'extinction de l'action publique ${ }^{43}$.

À l'occasion de sa décision rendue le 4 novembre 2016, le droit au silence ou droit de se taire dans le cadre d'une procédure pénale a été pour la première fois reconnu expressément comme ayant un caractère constitutionnel ${ }^{44}$. Selon le Code de procédure pénale, l'obligation de prêter serment n'est pas applicable aux personnes placées en garde à vue. Toutefois, selon la même disposition, "[l]e fait que les personnes gardées à vue aient été entendues après avoir prêté serment ne constitue [...] pas une cause de nullité de la procédure ${ }^{45}$. C'est sur ce dernier point que le Conseil a rendu une décision de censure. En effet,

Faire ainsi prêter serment à une personne entendue en garde à vue de «dire toute la vérité, rien que la vérité» peut être de nature à lui laisser croire qu'elle ne dispose pas du droit de se taire ou de nature à contredire l'information qu'elle a reçue concernant ce droit ${ }^{46}$.

Il y a donc méconnaissance du droit au silence dans le cadre d'une procédure pénale, qui découle du droit de ne pas s'accuser consacré par l'article 9 de la DDHC. Cependant, le commentaire de la décision apporte deux précisions quant à la portée de cette décision ${ }^{47}$. D'abord, «cette censure ne saurait signifier l'illégalité des auditions d'une personne entendue d'abord comme témoin puis placée en garde à vue ${ }^{48}$. Ensuite, le Conseil précise que ce sera «au juge judiciaire de déterminer au cas par cas [...] les conséquences à tirer d'une telle irrégularité ${ }^{49}$.

Protectrice des droits et libertés constitutionnels en matière pénale, la jurisprudence du Conseil en 2016 n'est toutefois pas exempte de critiques. En effet, le Conseil constitutionnel a rendu plusieurs décisions contestables, en particulier en matière économique et fiscale.

Le Conseil a déclaré conformes à la Constitution des dispositions subordonnant la mise en mouvement de l'action publique pour la répression de certaines infractions fiscales, notamment celle de délit de fraude fiscale ${ }^{50}$, au dépôt d'une plainte préalable par l'administration. C'est ce que l'on appelle le "verrou de Bercy». Dans une décision rendue le 22 juillet 2016, le Conseil a estimé que si ces dispositions constituaient bien une atteinte au libre exercice de l'action publique par le procureur de la République, celle-ci n'était toutefois pas «disproportionnée ${ }^{51}$. Il n'y a donc pas, entre autres, méconnaissance du principe d'indépendance de l'autorité judiciaire et de séparation des pouvoirs. En effet, l'atteinte n'est pas disproportionnée selon le Conseil pour un certain nombre de raisons ${ }^{52}$. Il considère que la règle est justifiée par la nature des infractions concernées qui

[...] portent atteinte aux intérêts financiers de l'État et causent un préjudice principalement au Trésor public. Ainsi, en l'absence de dépôt d'une plainte de l'administration, à même d'apprécier la gravité des atteintes portées à ces intérêts collectifs protégés par la loi fiscale, qui sont susceptibles de faire l'objet de sanctions administratives, l'absence de mise en mouvement de l'action publique ne constitue pas un trouble substantiel à l'ordre public ${ }^{53}$.

Le Conseil n'effectue donc ici qu'un contrôle restreint $^{54}$. Or,

[...] n'est-ce pas le cœur des fonctions du parquet économique et financier de repérer les cas les plus graves avec de véritables schémas de fraudes très souvent liés à d'autres infractions? Ou encore de soulever des dossiers sensibles [...] en échappant à la sphère politique du fait précisément de son indépendance ${ }^{55}$ ?

L'argumentation retenue par le Conseil pour déclarer conforme à la Constitution ce dispositif semble un peu

42. Commentaire de la décision no 2016-569 QPC, p. 11, en ligne: http://www.conseil-constitutionnel.fr/conseil-constitutionnel/root/bank/ download/2016569QPC2016569qpc_ccc.pdf.

43. Ibid. Ce n'est pas la première fois que le Conseil considère que les droits de la défense «ne se limitent pas aux seules sanctions ayant le caractère de punition» (ibid.).

44. Commentaire de la décision no 2016-594 QPC du 4 novembre 2016, $M^{\text {me }}$ Sylvie T., p. 13, en ligne: http://www.conseil-constitutionnel.fr/conseilconstitutionnel/root/bank/download/2016594QPC20165949pc_ccc.pdf.

45. Art. 153 du Code de procédure pénale dans sa rédaction issue de la loi no 2004-204 du 9 mars 2004 portant adaptation de la justice aux évolutions de la criminalité, Journal officiel de la République française, n 59,10 mars 2004, p. 4567.

46. CC, déc. n 2016-594 QPC, $\$ 8$.

47. Voir F. Malhiere, «La constitutionnalisation du droit au silence dans le cadre d'une procédure pénale», Gazette du Palais, n 11,14 mars 2017 , p. 32: «Si la pédagogie et le réalisme de cette motivation sont à saluer, la portée de la censure mérite d'être précisée».

48. Commentaire de la décision $\mathrm{n}^{\circ}$ 2016-594 QPC, p. 15.

49. Ibid.

50. Le champ d'application de cette dérogation est large; voir le commentaire de la décision no 2016-555 QPC du 22 juillet 2016 , M. Karim B., p. 5, en ligne: http://www.conseil-constitutionnel.fr/conseil-constitutionnel/root/bank/download/2016555QPC2016555qpc_ccc.pdf.

51. CC, déc. n 2016-555 QPC, \$15.

52. Ibid., $\$ 12$ et 14 .

53. Ibid., $\$ 13$.

54. Voir A.-L. Cassard-Valembois, «Le “verrou de Bercy” n’a pas sauté!», L'actualité juridique. Droit administratif, 2016, p. 1925 sq.

55. M.-E. Boursier, «Verrou de Bercy: consécration manquée du principe d'indépendance du Parquet», L'actualité juridique. Droit pénal, 2016, p. 538. 
légère ${ }^{56}$ au regard des critiques dont il fait l'objet ${ }^{57}$. En outre, cette décision «marque la rétrogradation du principe de la séparation des pouvoirs dans le contentieux de la QPC ${ }^{58}$, celui-ci devant désormais, pour faire l'objet d'une QPC, être invoqué «dans le cas où cette méconnaissance [du principe de séparation des pouvoirs] affecte par elle-même un droit ou une liberté que la Constitution garantit ${ }^{59}$.

Le Conseil constitutionnel s'est également prononcé à plusieurs reprises en 2016 en matière de cumul des poursuites et de sanctions. Si sa jurisprudence est bien établie s'agissant du cumul de sanctions ${ }^{60}$, tel n'est pas le cas de celle relative au cumul des poursuites.

Dans sa décision remarquée du 18 mars 2015, le Conseil constitutionnel a déclaré contraire au principe de nécessité des délits et des peines, et non au regard de l'adage «non bis in idem ${ }^{61}$, le cumul des poursuites pour délit d'initié et pour manquement d'initié. Il a modifié son considérant de principe:

[...] les principes ainsi énoncés [principes de légalité et de nécessité des délits et des peines] ne concernent pas seulement les peines prononcées par les juridictions pénales mais s'étendent à toute sanction ayant le caractère d'une punition $^{62} ;[\ldots]$ le principe de nécessité des délits et des peines ne fait pas obstacle à ce que les mêmes faits commis par une même personne puissent faire l'objet de poursuites différentes aux fins de sanctions de nature administrative ou pénale en application de corps de règles distincts devant leur propre ordre de juridiction ${ }^{63}$.

Pour qu'un tel cumul soit admis, il faut «qu'au moins l'une des conditions suivantes soit remplie ${ }^{64}$ : les dispositions ne doivent pas réprimer les mêmes faits; elles ne doivent pas protéger les mêmes intérêts sociaux; elles doivent aboutir au prononcé de sanctions de nature différente; les poursuites et sanctions ne doivent pas relever du même ordre de juridiction ${ }^{65}$. A contrario, pour que le cumul des poursuites soit considéré comme inconstitutionnel, ce qui était le cas en l'espèce, il faut qu'il existe « une quadruple identité: de fait, d'intérêt social protégé, de nature des sanctions et d'ordre juridictionnel ${ }^{66}$. L'appréciation de ces différents critères paraît toutefois relativement subjective, laissant ainsi au Conseil une marge d'appréciation non négligeable.

En 2016, si le Conseil a développé sa jurisprudence en la matière, il ne l'a pour autant pas rendue plus lisible, bien au contraire. Dans sa décision du 14 janvier 2016, il s'est prononcé sur la conformité au principe de nécessité des délits et des peines des dispositions prévoyant le cumul de poursuites en matière de délit et de manquement d'initié, mais dans des rédactions issues d'autres lois que celles censurées par le Conseil en mars $2015^{67}$. Il ne retient toutefois pas la même solution puisqu'il les déclare conformes. Confirmant sa jurisprudence, le Conseil relève que le cumul des poursuites était bien possible puisque «les faits prévus et réprimés par les articles précités doivent être regardés comme susceptibles de faire l'objet de sanctions de nature différente ${ }^{68}$. Pour en arriver à cette conclusion, le Conseil compare les sanctions encourues: le manquement d'initié et le délit d'initié sont tous deux passibles d'une amende d'1500 ooo euros. Toutefois,

[...] le juge pénal peut condamner l'auteur d'un délit d'initié à une peine d'emprisonnement lorsqu'il s'agit d'une personne physique; [...] d'autre part, lorsque l'auteur d'un délit d'initié est une personne morale, le

56. Dans une autre décision relative au gel des avoirs en matière de terrorisme, on peut également regretter que le Conseil ait été avare de précisions dans la rédaction de ses motifs, notamment celui relatif à la présomption d'innocence. Voir CC, déc. no 2015-524 QPC du 2 mars 2016, M. Abdel Manane M. K. ; C. Mauro, "Le gel d'avoirs n'est pas une sanction... mais un peu quand même», La semaine juridique, édition générale, n 20-21, 2016, 589 .

57. Parmi les critiques, on peut retenir par exemple le fait que ce verrou « renforce de façon excessive un pouvoir discrétionnaire de l'administration fiscale qui est soumis à bien d'autres impératifs que la punition du fraudeur, ce qui aboutit à des inégalités très fâcheuses des fraudeurs devant la loi pénale" (C. David, Les grands arrêts de la jurisprudence fiscale, $5^{\mathrm{e}}$ éd., Paris, Dalloz, 2009; cité par A.-L. Cassard-Valembois, «Le "verrou de Bercy" n’a pas sauté!»); il faut préciser ici qu'en raison de la délimitation du champ de la QPC, c'est le principe de ce verrou qui est déclaré conforme à la Constitution et non ses conditions d'exercice, cette reconnaissance «semble donc virtuelle» (ibid.).

58. A.-L. Cassard-Valembois, «Le "verrou de Bercy" n'a pas sauté!».

59. CC, déc. n 2016-555 QPC, \$9; voir A.-L. Cassard-Valembois, «Le “verrou de Bercy” n’a pas sauté!».

60. CC, déc. $\mathrm{n}^{\circ}$ 82-143 DC du 30 juillet 1982, Loi sur les prix et les revenus, cons. 13: «[...] la règle du non-cumul des peines en matière de crimes et délits $[\ldots]$ n'a que valeur législative et [...] il peut donc toujours y être dérogé par une loi »; le Conseil effectue un contrôle de proportionnalité : celui-ci «implique, qu'en tout état de cause, le montant global des sanctions éventuellement prononcées ne dépasse pas le montant le plus élevé de l'une des sanctions encourues" (voir par exemple CC, déc. n 89-26o DC du 28 juillet 1989, Loi relative à la sécurité et à la transparence du marché financier, cons. 22).

61. C'est au regard de l'article 8 de la DDHC que le Conseil a censuré les dispositions législatives contestées, et non au regard de l'adage «non bis in idem». Implicitement, cet adage apparaît donc dépourvu de valeur constitutionnelle; voir J. Bossan, «Le cumul des poursuites appréhendé par le Conseil constitutionnel ", Actualité juridique. Droit pénal, 2015, p. 179 sq.

62. Les mesures de faillite et d'interdiction prononcées par le juge civil ou commercial dans le cadre d'un redressement ou d'une liquidation judiciaire ont bien le caractère d'une punition (CC, déc. $\mathrm{n}^{\circ}$ 2016-570 QPC du 29 septembre 2016, M. Pierre M. et CC, déc. n 2016-573 QPC du 29 septembre 2016, M. Lakhdar Y.).

63. CC, déc. $\mathrm{n}^{\circ}$ 2014-453/454 QPC et 2015-462 QPC du 18 mars 2015, M. John L. et autres, cons. 19.

64. Commentaire des décisions n 2014-453/454 QPC et 2015-462 QPC, p. 10, en ligne: http://www.conseil-constitutionnel.fr/conseil-constitutionnel/ root/bank/download/2014453_454QPCet2015462QPC2014453_454_462qpc_ccc.pdf.

65. CC, déc. no 2014-453/454 QPC et 2015-462 QPC, cons. 24 à 27.

66. O. Décima, «Tombeau de ne bis in idem», Recueil Dalloz, 2016, p. 931 sq.

67. CC, déc. $\mathrm{n}^{\circ} 2015-513 / 514 / 526$ QPC.

68. Ibid., cons. 12. 
juge pénal peut prononcer sa dissolution et une amende cinq fois supérieure à celle pouvant être prononcée par la commission des sanctions de l'Autorité des marchés financiers $[\ldots]^{69}$.

Pour départager les sanctions qui sont de même nature et celles qui sont de nature différente, le Conseil ne s'appuie pas sur l'objet, à savoir le fait que la peine encourue soit une peine d'emprisonnement ou une peine d'amende. C'est le critère de la gravité qui est utilisé ${ }^{70}$ : il examine si les sanctions «sont d'une sévérité comparable ${ }^{71}$, ce qui n'était pas le cas en l'espèce des amendes encourues pour manquement d'initié qui peuvent être très importantes et de la possibilité pour le juge pénal de condamner l'auteur du délit d'initié à une peine d'emprisonnement. Dans deux décisions du 29 septembre 2016, il a également considéré que les sanctions prévues par le Code de commerce en cas de redressement judiciaire et en cas de banqueroute étaient de nature différente, ne faisant ainsi pas obstacle au cumul des poursuites au sens de l'article 8 de la $\mathrm{DDHC}^{72}$. En effet, si le juge pénal peut prononcer une peine d'emprisonnement, une peine d'amende et d'autres peines complémentaires, le juge civil ou commercial ne peut que prononcer la faillite et les interdictions prévues par l'article L. 653-8 du Code du commerce ${ }^{73}$. Toutefois, dans sa décision du 30 septembre 2016, le Conseil a considéré que les sanctions prévues par le Code monétaire et financier pour réprimer le manquement de diffusion de fausses informations et celui de délit de diffusion de fausses informations étaient, au contraire, de même nature ${ }^{74}$. Dans le commentaire de cette décision, il est précisé qu'en l'espèce,

[...] dès lors qu'il avait jugé dans la décision du 18 mars 2015 que l'importance de l'amende encourue [celle-ci était de 10 millions d'euros] était d'une sévérité équivalente à la peine de deux ans d'emprisonnement pour le délit d'initié, il a jugé qu'il en était de même dans l'hypothèse dont il était saisi [la peine encourue en l'espèce pour manquement d'initié peut atteindre 100 millions d'euros] ${ }^{75}$.
Ainsi, «[i]l serait aussi désagréable de passer deux ans en prison [...] que de payer cent millions d'euros d'amende ${ }^{76}$. Le Conseil adopte finalement une jurisprudence peu claire, notamment sur ce critère de l'identité de la nature des sanctions, qui lui laisse, là encore, une large marge d'appréciation ${ }^{77}$.

Dans deux décisions rendues cette fois en matière fiscale, le Conseil a utilisé un raisonnement pour le moins surprenant. Les requérants, dont l'ancien ministre Jérôme Cahuzac, espéraient que le Conseil prononce l'inconstitutionnalité des dispositions du Code général des impôts permettant un cumul entre des pénalités fiscales et des sanctions pénales. Il fallait donc, en principe, démontrer que les critères dégagés en mars 2015 étaient bien remplis, c'est-à-dire qu'il y avait bien une identité sur les quatre points. Pourtant, le Conseil ne va pas appliquer sa jurisprudence. Il la rappelle, mais dans une version allégée puisqu'il supprime un des critères, celui de l'ordre de juridiction. Désormais, le «fait que les poursuites et les sanctions prononcées relèvent ou non du même ordre juridictionnel est [...] indifférent pour l'exercice du contrôle effectué par le Conseil constitutionnel ${ }^{78}$. Toutefois, "afin de mieux contourner le problème et d'éluder l'application du principe de nécessité des délits et des peines», «le Conseil constitutionnel s'affranchit de sa méthode pour jouer sur un autre registre, inédit celui-là : la complémentarité des sanctions pénale et fiscale ${ }^{79}$.

Le Conseil n'examine pas s'il y a en l'espèce triple identité entre les deux procédures. Il considère que les articles 1729 et 1741 du Code général des impôts forment un «ensemble ${ }^{80}$. Celui-ci vise à assurer :

[...] la protection des intérêts financiers de l'État ainsi que l'égalité devant l'impôt, en poursuivant des finalités communes, à la fois dissuasive et répressive. Le recouvrement de la nécessaire contribution publique et l'objectif de lutte contre la fraude fiscale justifient l'engagement de procédures complémentaires dans les cas de fraudes les plus graves ${ }^{81}$.

Il ne s'agit pas de "poursuites différentes», il n'y a donc pas lieu pour le Conseil d'appliquer sa jurisprudence

69. CC, déc. $\mathrm{n}^{\circ} 2015-513 / 514 / 526$ QPC.

70. «L'amende et l'emprisonnement peuvent donc avoir la même "nature", comme cela a déjà été admis dans la décision du 18 mars 2015 , alors qu'inversement, des sanctions dont la matérialité est identique peuvent être de "nature différente" " (O. Decima, "Tombeau de ne bis in idem»)

71. Commentaire des décisions n 2016-570 QPC et n 2016-573 QPC, p. 22, en ligne: http://www.conseil-constitutionnel.fr/conseil-constitutionnel/ root/bank/download/2016570QPC2016570qpc_ccc.pdf.

72. CC, déc. n 2016-570 QPC et 2016-573 QPC.

73. CC, déc. $\mathrm{n}^{\circ}$ 2016-570 QPC, $\$ 7$ et 8 .

74. CC, déc. $n^{\circ}$ 2016-572 QPC du 30 septembre 2016, M. Gilles $M$. et autres, $\$ 12$; si les trois critères d'identité étaient réunis en l'espèce, le juge n’a pas déclaré contraire au principe de nécessité des délits et des peines ce cumul. Il a émis une réserve dans son $\$ 16$, en prenant en compte l'intervention du législateur; voir J.-H. Robert, «Le Conseil constitutionnel rend une nouvelle décision de règlement à propos du cumul de poursuites contre les abus de marché», La semaine juridique, édition générale, $\mathrm{n}^{\circ} 49,2016,1315$.

75. Commentaire de la décision $n^{\circ}$ 2016-572 QPC, p. 16, en ligne: http://www.conseil-constitutionnel.fr/conseil-constitutionnel/root/bank/ download/2016572QPC2016572qpc_ccc.pdf.

76. J.-H. Robert, «Le Conseil constitutionnel rend une nouvelle décision...».

77. Voir A.-V. Le Fur, D. Schmidt, «Le traitement du cumul des sanctions administratives et pénales en droit interne: entre incohérences et insécurité juridique», Recueil Dalloz, 2016, p. 2126.

78. Commentaire des décisions $\mathrm{n}^{\circ}$ 2016-570 QPC et 2016-573 QPC, p. 19.

79. C. Mandon, «La guerre contre la fraude fiscale aura bien lieu, commentaire des décisions QPC no $2016-545$ et 546 », Constitutions, 2016 , p. 436 sq.

80. CC, déc. $\mathrm{n}^{\circ}$ 2016-546 QPC du 24 juin 2016, M. Jérôme C., \$20.

81. Ibid. (nous soulignons). 
relative au cumul des poursuites ${ }^{82}$. Il a toutefois émis des réserves d'interprétation, notamment une dans laquelle il considère que le principe de nécessité des délits et des peines «impose néanmoins que les dispositions de l'article 1741 ne s'appliquent qu'aux cas les plus graves de dissimulation frauduleuse de sommes soumises à l'impôt ${ }^{83}$. Dès lors, seuls les manquements les plus graves pourront faire l'objet de ces deux "procédures complémentaires » prévues aux articles 1729 et 1741 . Le Conseil n'hésite pas à préciser ce qu'il faut entendre par ces «cas les plus graves» qui peuvent résulter « du montant des droits fraudés, de la nature des agissements de la personne poursuivie ou des circonstances de leur intervention ${ }^{84}$. La liberté prise par le Conseil est grande ${ }^{85}$, liberté dont le service juridique du Conseil semble avoir conscience puisque le commentaire de la décision paraît chercher à justifier la décision adoptée et précise que le Conseil n'est «pas allé au-delà de celle-ci [la volonté du législateur] dans la formulation de sa réserve d'interprétation ${ }^{86}$.

Enfin, dans une décision du $1^{\text {er }}$ juillet 2016, le Conseil s'est prononcé sur une disposition qui «se contente d'énoncer le principe d'un possible cumul de poursuites pénales et de poursuites devant la CDBF [Cour de discipline budgétaire et financière] ${ }^{87}$. Alors qu'en mars 2015, le «Conseil était [...] saisi de la constitutionnalité de texte définissant spécifiquement les infractions de délit d'initié et de manquement d'initié», en l'espèce, «l'article soumis à l'examen du Conseil ne définit aucune infraction susceptible de donner lieu à une double procédure; il se borne à la rendre possible en général ${ }^{88}$. Le Conseil déclare donc cet article conforme à la Constitution, sous réserve que soient respectés, concernant le cumul de poursuites, le principe de nécessité des délits et des peines ${ }^{89}$, et concernant le cumul de sanctions, le principe de proportionnalité $^{90}$. Ainsi, le Conseil laisse la porte ouverte s'agissant des dispositions plus précises qui prévoient les différentes infractions susceptibles d'occasionner un cumul $^{91}$. Ces dispositions relatives à l' «obscure» Cour de discipline budgétaire et financière devraient donc probablement revenir rapidement rue de Montpensier.

\section{Le renforcement des droits des collectivités}

Saisi par le Conseil d'État, le Conseil constitutionnel a eu à se prononcer dans la décision n ${ }^{\circ}$ 2016-565 QPC du 16 septembre 2016 d'une QPC posée pour l'Assemblée des départements de France relative à la conformité constitutionnelle de l'article L. 3211-1 du Code général des collectivités territoriales, dans sa rédaction résultant de la loi no $2015-991$ du 7 août 2015 portant nouvelle organisation territoriale de la République. La question posée portait plus précisément sur les mots «dans les domaines de compétences que la loi lui attribue ${ }^{92}$. En effet, l'association requérante estimait qu'en supprimant la clause dite « de compétence générale ${ }^{93}$ reconnue aux départements, sans prévoir de dispositif leur permettant d'intervenir dans les domaines pour lesquels aucune autre personne publique ne dispose d'une compétence attribuée par la loi, le législateur a méconnu le principe de libre administration des collectivités territoriales. Au soutien de sa requête, l'association se référait à la décision du Conseil constitutionnel no 2010-618 DC du 9 décembre 2010 qui en aurait alors fait une condition du respect du principe invoqué. Le Conseil considère toutefois que la suppression de ladite clause n'est pas contraire au principe de libre administration des collectivités territoriales. Après avoir considéré que ce principe n'implique pas que «les collectivités territoriales doivent pouvoir intervenir dans les domaines pour lesquels aucune autre personne publique ne dispose d'une compétence attribuée par la loi » ${ }^{94}$, il affirme que

[...] compte tenu de l'étendue des attributions dévolues aux départements par les dispositions législatives en vigueur, qu'il s'agisse de compétences exclusives, de compétences partagées avec d'autres catégories de collectivités territoriales ou de compétences susceptibles d'être déléguées par d'autres collectivités territoriales, les dispositions contestées ne privent pas les départements d'attributions effectives ${ }^{95}$.

Il explicite ainsi le principe en en donnant une interprétation assez restrictive et surtout il ne l'interprète pas à la lumière du principe de subsidiarité comme il l'avait pourtant fait dans sa décision $n^{\circ} 2010-618$ DC.

82. Commentaire des décisions no 2016-545 QPC du 24 juin 2016, M. Alec W. et autre et 2016-546 QPC du 24 juin 2016 , M. Jérôme C., p. 25, en ligne: http://www.conseil-constitutionnel.fr/conseil-constitutionnel/root/bank/download/2016546QPC2016546qpc_ccc.pdf; voir C. Mandon, «La guerre contre la fraude fiscale...».

83. CC, déc. $\mathrm{n}^{\circ} 2016-546$ QPC, $\$ 21$.

84. Ibid.

85. Pour une critique de ces décisions, voir A.-V. Le Fur, D. Schmidt, «Le traitement du cumul des sanctions...».

86. Commentaire des décisions $\mathrm{n}^{\circ}$ 2016-545 QPC et 2016-546 QPC, p. 25.

87. Commentaire de la décision n ${ }^{\circ}$ 2016-550 QPC du $1^{\text {er }}$ juillet 2016, M. Stéphane R. et autre, p. 7, en ligne: http://www.conseil-constitutionnel.fr/ conseil-constitutionnel/root/bank/download/2016550QPC2016550qpc_ccc.pdf.

88. F. Waserman, «L'application du principe non bis in idem et les procédures devant la CDBF», Constitutions, 2016, p. 456 sq.

89. CC, déc. $\mathrm{n}^{\circ}$ 2016-550 QPC, $\$ 7$.

90. Ibid., $\$ 8$.

91. Voir F. Waserman, «L'application du principe non bis in idem...»; O. Le Bot, "Cumul de sanctions pénales et disciplinaires: deux réserves d'interprétation pour la CDBF", Constitutions, 2016, p. 468-470. Le Conseil en l'espèce émet toutefois deux réserves d'interprétation, au regard du principe de nécessité des délits et des peines; voir CC, déc. nº 2016-550 QPC, $\$ 7$ et 8.

92. CC, déc. n 2016-565 QPC du 16 septembre 2016, Assemblée des départements de France, $\$ 1$.

93. Ibid., $\$ 2$.

94. Ibid., $\$ 5$.

95. Ibid., $\$ 6$ 
Dans ce sens d'une interprétation relativement restrictive du principe de libre administration des collectivités territoriales, on relève également la décision $\mathrm{n}^{\circ}{ }^{2016-}$ 597 QPC, que le Conseil rend le 25 novembre 2016. Était en cause l'article L. 4424-9 du Code général des collectivités territoriales relatif au plan d'aménagement et de développement durable de Corse. Il y est précisé que la collectivité territoriale de Corse fixe l'échelle des documents cartographiques annexés au plan d'aménagement et la localisation des sites remarquables. La commune requérante invoquait le non-respect du principe de libre administration des collectivités territoriales ainsi que du principe d'interdiction de la tutelle d'une collectivité territoriale sur une autre. Le Conseil constitutionnel a pourtant jugé de la conformité de la disposition à la Constitution considérant que seul un rapport de comptabilité et non de conformité existait entre les documents d'urbanisme et le plan d'aménagement.

Dans sa décision n ${ }^{\circ}$ 2016-588 QPC du 21 octobre 2016, le Conseil s'est prononcé sur la constitutionnalité du paragraphe II de l'article L. 2113-5 du Code général des collectivités territoriales relatif aux conséquences de la création d'une commune nouvelle sur les établissements publics de coopération intercommunale à fiscalité propre auxquels appartenaient précédemment les communes fusionnées. Cette disposition issue de la loi NOTRe ${ }^{96}$ méconnaîtrait le principe de libre administration des collectivités territoriales dans la mesure où elle autorise le préfet à imposer à la commune nouvelle, lors de sa création, son rattachement à un établissement public de coopération intercommunale à fiscalité propre autre que celui en faveur duquel elle se serait prononcée. Il est également invoqué une atteinte au principe d'égalité étant considéré que la disposition contestée traite différemment les communes en fonction de leur appartenance ou non à certaines intercommunalités. Le Conseil admet dans un premier temps que la disposition en cause affecte le principe de libre administration des communes mais en considérant dans le même temps que le législateur a poursuivi un but d'intérêt général dans la mesure où il «a entendu éviter que son choix puisse porter atteinte à la cohérence ou à la pertinence des périmètres intercommunaux existants ${ }^{97}$. Toutefois, dans un second temps, il juge qu'il est porté une atteinte manifestement disproportionnée audit principe. Au regard des conséquences que le rattachement à un établissement public de coopération intercommunale à fiscalité propre a nécessairement pour la commune nouvelle, il considère que la loi devrait prévoir la consultation de l'organe délibérant de l'établissement public de coopération intercommunale à fiscalité propre auquel le rattachement est envisagé. Devrait également être prévue la consultation des organes délibérants des établissements publics de coopération intercommunale à fiscalité propre dont la commune nouvelle est susceptible de se retirer ainsi que celle des conseils municipaux des communes membres de ces établissements publics. De même, en cas de désaccord avec le projet de rattachement, ces établissements publics et ces communes doivent pouvoir provoquer la saisine de la commission départementale de coopération intercommunale ${ }^{98}$. Le Conseil décide en conséquence de l'abrogation des dispositions contestées, mais il en reporte les effets au 31 mars 2017 afin de permettre au législateur d'apprécier les conséquences qu'il convient de tirer de cette déclaration d'inconstitutionnalité. En effet, en l'espèce, l'abrogation immédiate rendrait impossible la détermination de l'établissement public de coopération intercommunale à fiscalité propre auquel la commune pourrait se rattacher en cas de fusion de plusieurs communes membres d'établissements publics de coopération intercommunale à fiscalité propre distincts. Le Conseil constitutionnel précise par ailleurs que, dans un souci de préservation de l'effet utile de sa décision,

[...] la déclaration d'inconstitutionnalité peut être invoquée dans les instances en cours ou à venir dont l'issue dépend de l'application des dispositions déclarées inconstitutionnelles.

\section{Et qu'}

En cas d'annulation, sur ce fondement, de l'arrêté préfectoral portant rattachement d'une commune nouvelle à un établissement public de coopération intercommunale à fiscalité propre, les deux dernières phrases du troisième alinéa du paragraphe II de l'article L. 2113-5 du code général des collectivités territoriales sont applicables ${ }^{99}$.

Le Conseil constitutionnel a été saisi par le Conseil d'État d'une QPC relative aux $1^{\circ}, 2^{\circ}$ et $3^{\circ}$ du paragraphe I de l'article 104 de la loi n ${ }^{\circ}$ 2007-1824 du 25 décembre 2007 de finances rectificative pour 2007 . Il a rendu la décision $\mathrm{n}^{\mathrm{o}}$ 2016-549 QPC, le $1^{\mathrm{er}}$ juillet 2016. La collectivité de SaintMartin faisait valoir que les ressources qui lui avaient été attribuées en application de ces dispositions étaient insuffisantes au regard des charges résultant des compétences de la commune de Saint-Martin qui lui avaient été transférées. Le Conseil a d'abord jugé que la compensation financière des charges résultant des compétences transférées était assurée et que les dispositions contestées n'avaient pas pour effet de réduire le montant des ressources propres de la collectivité de Saint-Martin. Les griefs tirés de la méconnaissance des principes de libre administration et d'autonomie financière ne pouvaient donc qu'être rejetés. Le Conseil a ensuite jugé qu'il n'incombait pas au législateur de garantir une compensation intégrale des charges résultant des transferts de compétences entre collectivités. Il a donc écarté le grief tiré de la méconnaissance du principe de compensation des charges résultant de tels transferts de compétence. Le

96. Loi no $2015-991$ du 7 août 2015 portant nouvelle organisation territoriale de la République.

97. CC, déc. $n^{\circ}$ 2016-588 QPC du 21 octobre 2016, Communauté de communes des sources du lac d'Annecy et autre, $\$ 8$.

98. Ibid., \$9.

99. Ibid., $\$ 13$. 
Conseil a en conséquence déclaré conformes à la Constitution les dispositions des $1^{\circ}, 2^{\circ}$ et $3^{\circ}$ du paragraphe I de l'article 104 de la loi du 25 décembre 2007.

\section{La protection par le Conseil des droits économiques et sociaux: l'art de ménager la chèvre et le chou}

\section{A. Les droits des travailleurs: une jurisprudence en demi-teinte}

Dans sa jurisprudence de 2016, le Conseil a été amené à plusieurs reprises à se prononcer sur la constitutionnalité de dispositions à teneur sociale. Plusieurs lois ont en effet été adoptées récemment avec pour objectif de «moderniser» le modèle social existant. Les années 2015 et 2016 sont d'ailleurs considérées comme étant la période centrale du quinquennat de François Hollande pour les réformes socio-économiques qui ont été menées ${ }^{100}$ : loi $\mathrm{n}^{\circ}$ 2016-41 du 27 janvier 2016 de modernisation de notre système de santé, loi n ${ }^{\circ}$ 2016-1088 du 8 août 2016 relative au travail, à la modernisation du dialogue social et à la sécurisation des parcours professionnels, loi no 2016-1547 du 19 novembre 2016 de modernisation de la justice du $\mathrm{XXI}^{\mathrm{e}}$ siècle, loi $\mathrm{n}^{\circ}$ 2016-1691 du 10 décembre 2016 relative à la transparence, à la lutte contre la corruption et à la modernisation de la vie économique.

Au-delà des lois nouvelles contrôlées par le Conseil, plusieurs saisines par la voie de la QPC l'ont conduit à résoudre certains contentieux sociaux issus, le plus souvent, de procédures de licenciement individuel ou collectif. Le Conseil a notamment été sollicité pour trancher le conflit opposant la société Goodyear Dunlop à ses salariés ${ }^{101}$. En 2014, la société mère américaine Goodyear, après avoir quadruplé ses bénéfices ${ }^{102}$, invitait sa filiale française à procéder au licenciement économique de plus de 1000 salariés. 722 d'entre eux décidèrent de saisir le conseil des prud'hommes d'Amiens en vue d'être indem- nisés pour licenciements sans cause réelle et sérieuse. La société Goodyear Dunlop souleva l'inconstitutionnalité des dispositions législatives prévoyant l'obligation pour les entreprises de plus de onze salariés d'indemniser d'un montant au moins égal aux six derniers mois de salaire ceux ayant plus d'un an d'ancienneté qui sont licenciés sans cause réelle et sérieuse ${ }^{103}$. Selon la société requérante, l'article L. 1235-3, alinéa 2 du Code du travail ainsi contesté serait contraire à la liberté d'entreprendre et constituerait une rupture d'égalité avec les entreprises de moins de onze salariés qui n'ont pas à respecter le plancher des six mois de salaire. Le Conseil va rejeter les deux arguments. Concernant la liberté d'entreprendre, il va estimer qu'

[...] en visant à dissuader les employeurs de procéder à des licenciements sans cause réelle et sérieuse, les dispositions contestées mettent en œuvre le droit de chacun d'obtenir un emploi découlant du cinquième alinéa du Préambule de la Constitution de 1946. En prévoyant une indemnité minimale égale à six mois de salaire, ces dispositions ne portent pas une atteinte disproportionnée à la liberté d'entreprendre ${ }^{104}$

La solution dégagée en l'espèce s'inscrit dans une jurisprudence constante du Conseil qui a tendance à ne pas retenir l'atteinte à la liberté d'entreprendre. Ceci s'explique certainement par le fait que les lois de ces trente dernières années tendent davantage à lever les obstacles à la liberté économique qu'à en poser ${ }^{105} \mathrm{et}$, lorsqu'elles en admettent, c'est le plus souvent de manière circonspecte ${ }^{106}$.

Plus surprenant pouvait être perçu le développement suivi par le Conseil sur le principe d'égalité dans la mesure où il admet en l'espèce ce qu'il semblait avoir refusé dans une décision de 2015, à savoir l'utilisation par le législateur du critère de l'effectif dans l'entreprise pour fonder une différence de traitement dans l'indemnisation due aux salariés licenciés sans cause réelle et sérieuse. Une telle différence semblait avoir été bannie par le Conseil dans sa décision $\mathrm{n}^{\circ}$ 2015-715 DC du 5 août 2015 relative à la loi pour la croissance, l'activité et l'égalité des chances

100. Voir L. Daovannary, «Rétrospective 2015-2016, l'année des réformes structurantes», 22 juillet 2016, en ligne: http://www.editions-legislatives.fr/ content/r\% $3 \%$ A9trospective-2015-2016-lann\%C $3 \%$ A9e-des-r\% $\mathrm{C}_{3} \%$ A9formes-structurantes.

101. CC, déc. no 2016-582 QPC du 13 octobre 2016, Société Goodyear Dunlop Tires France SA.

102. «Le fabricant américain de pneus Goodyear a réalisé de gros bénéfices l'an dernier, grâce à un crédit d'impôt qui lui a permis de compenser des effets de change défavorables et une baisse des volumes de ventes en Europe. Sur l'ensemble de l'année 2014, le groupe d'Akron (Ohio, nord-est) a engrangé un bénéfice net de 2,45 milliards de dollars, soit plus que quadruplé comparé à 2013, a-t-il annoncé mardi. Cette année-là, le bénéfice net s'élevait à 600 millions de dollars» (AFP, "Goodyear a quadruplé son bénéfice en 2014», Le Figaro, 17 février 2015, en ligne: http://www. lefigaro.fr/flash-eco/2015/02/17/97002-20150217FILWWWoo213-goodyear-chiffre-d-affaires-en-baisse-au-t4.php).

103. Art. L. 1235-3, al. 1 et 2 du Code du travail.

104. CC, déc. $n^{\circ}$ 2016-582 QPC, $\$ 11$.

105. Voir en ce sens Jean Mouly qui rappelle que «l'idée de flexibilité» favorable à l'employeur est présente «dans nombre de dispositions récentes» permettant «d'alléger les contraintes de la loi pour une catégorie d'entreprises». Par ailleurs, l'auteur souligne que «la notion d'intérêt général, en droit du travail, est une notion de plus en plus évanescente, trop souvent instrumentalisée au profit de politiques de flexibilité néolibérales » (J. Mouly, «Le plancher d'indemnisation des licenciements injustifiés peut varier selon les effectifs de l'entreprise. Commentaire de la décision du Conseil constitutionnel, 13 octobre 2016, n 2016-582 QPC, Société Goodyear Dunlop Tires France», Droit social, 2016, p. 1066; contra P. Delvolvé, "Les libertés économiques», Revue française de droit administratif, 2017, p. 33 sq.).

106. Si les atteintes à la liberté d'entreprendre sont excessives et tendent à remettre en cause le modèle économique libéral dominant, le Conseil procédera à la censure de la loi, comme ce fut le cas pour la loi de modernisation sociale qui prévoyait l'interdiction des licenciements boursiers, c'est-à-dire des licenciements économiques pour les sociétés qui ne sont pas en difficulté particulière mais souhaitent seulement améliorer leur compétitivité (CC, déc. n² 2001-455 DC du 12 janvier 2002, Loi de modernisation sociale). 
économiques ${ }^{107}$. Le Conseil avait alors considéré que le législateur

[...] devait retenir des critères présentant un lien avec le préjudice subi par le salarié; [...] si le critère de l'ancienneté dans l'entreprise est ainsi en adéquation avec l'objet de la loi, tel n'est pas le cas du critère des effectifs de l'entreprise; [...] par suite, la différence de traitement instituée par les dispositions contestées méconnaît le principe d'égalité devant la loi ${ }^{108}$.

En 2016, le Conseil ne va pas suivre ce raisonnement. Pour autant, selon le commentaire officiel, il n'y a pas lieu de penser que les deux décisions se contredisent ${ }^{109}$. Dans sa décision de 2015, le Conseil devait se prononcer sur l'instauration d'un plafonnement de l'indemnité au détriment des personnes embauchées dans les entreprises de moins de onze salariés alors qu'en 2016 il devait statuer sur l'existence d'un plancher indemnitaire au profit du personnel des entreprises de plus de onze salariés. Dans un cas, la loi était donc défavorable aux salariés en limitant par un plafond leur indemnisation, ce que le Conseil a censuré. Dans l'autre cas, à l'inverse, la loi était propice aux salariés en obligeant l'employeur à les indemniser en respectant un seuil minimal de six mois de salaire, ce que le Conseil a validé. Les deux décisions apportent ainsi des solutions différentes sans pour autant s'opposer puisque, dans les deux cas, le Conseil s'assure que les salariés ne sont pas lésés.

Mais dans la décision de 2016, les dispositions relatives à l'institution d'un seuil indemnitaire ne constituent finalement un avantage social que dans les entreprises de plus de onze salariés. Le Conseil aurait pu ne pas s'attarder sur ce point, étant donné qu'en l'espèce il lui était demandé de se prononcer sur la présence opportune d'un seuil indemnitaire dans les entreprises dépassant les onze salariés et non sur l'absence d'un tel seuil dans les petites entreprises. Pourtant, le Conseil va se focaliser quasi exclusivement sur le sort de ces entreprises à effectif réduit pour constater l'absence d'atteinte au principe d'égalité. Ainsi, au lieu de montrer que la loi est fondée à offrir des garanties aux salariés des entreprises de plus de onze salariés pour protéger le droit de chacun d'obtenir un emploi, le Conseil va souligner que la loi est fondée à ne pas offrir une garantie équivalente dans les entreprises à effectif plus limité. Il affirme qu'
En prévoyant que le montant minimal de l'indemnité accordée par le juge en cas de licenciement sans cause réelle et sérieuse est applicable aux seuls licenciements dans les entreprises d'au moins onze salariés, le législateur a entendu éviter de faire peser une charge trop lourde sur les entreprises qu'il a estimées économiquement plus fragiles $[\ldots]^{110}$.

C'est finalement ce que l'on va retenir de cette décision et qui ressortira de la plupart des commentaires ${ }^{111}$. À Jean Mouly d'en conclure qu'

[...] une fois de plus, le Conseil constitutionnel se livre à une approche économique du texte contesté et raisonne en termes de coûts pour les entreprises, la pérennité des plus fragiles ne devant pas être menacée. Il faut donc bien reconnaître que, là encore, le Conseil tend à assimiler, à l'intérêt général, l'intérêt, sinon des entreprises dans leur ensemble, au moins d'une catégorie d'entre elles, les plus petites $^{112}$.

Au cours de la session 2016, le Conseil a eu l'occasion de rendre plusieurs autres décisions portant sur le droit du travail ${ }^{113}$ qui, comme la précédente, peuvent être considérées comme étant à la fois favorables et défavorables aux salariés, ou plus exactement, leur étant favorables seulement en apparence. Il s'est notamment prononcé à deux reprises sur la situation particulière des salariés licenciés pour faute lourde ${ }^{114}$, faute d'une particulière gravité revêtant un caractère intentionnel ${ }^{115}$.

Le Conseil a été saisi d'une première affaire soulevant l'inconstitutionnalité d'une disposition législative mettant à l'écart du bénéfice de l'indemnité compensatrice de congés payés les salariés dont la rupture du contrat de travail est inhérente à une faute lourde ${ }^{116}$. Le requérant estimait que cette privation était constitutive d'une violation du droit au repos, du droit à la protection de la santé et d'une atteinte au principe d'individualisation des peines. Mais le Conseil va préférer se prononcer sur un autre moyen, le principe d'égalité, dont il s'est saisi d'office.

On aurait pu s'attendre à ce que le Conseil analyse la potentielle rupture d'égalité au regard de la différence de traitement qui existe entre les salariés bénéficiaires de l'indemnité compensatrice de congés payés et ceux qui, auteurs d'une faute lourde, en sont en principe

107. CC, déc. no 2015-715 DC du 5 août 2015, Loi pour la croissance, l'activité et l'égalité des chances économiques: Constitutions, 2015, p. 421-432, comm. A. Fabre

108. CC, déc. n 2015-715 DC, cons. 152.

109. Commentaire de la décision $n^{\circ}$ 2016-582 QPC, p. 9-10, en ligne: http://www.conseil-constitutionnel.fr/conseil-constitutionnel/root/bank/ download/2016582QPC2016582qpc_ccc.pdf.

110. CC, déc. $n^{\circ} 2016-582$ QPC, $\$ 8$.

111. Voir notamment le commentaire de la décision no 2016-582 QPC; J. Mouly, «Le plancher d'indemnisation...».

112. J. Mouly, «Le plancher d'indemnisation...», p. 1066

113. Voir notamment en plus des décisions ici étudiées: CC, déc. $\mathrm{n}^{\circ}$ 2016-533 QPC du 14 avril 2016, M. Jean-Marc P.

114. CC, déc. $\mathrm{n}^{\circ}$ 2015-523 QPC du 2 mars 2016, M. Michel O. : J. Mouly, «Invalidation par le Conseil constitutionnel des dispositions subordonnant le droit à l'indemnité compensatrice de congés payés à l'absence de faute lourde du salarié», Droit social, 2016, p. 475-478; CC, déc. n 2016558/559 QPC du 29 juillet 2016, M. Joseph L. et autre.

115. La Cour de cassation retient que la faute lourde est notamment constituée par la volonté malveillante du salarié qui cherche à nuire à l'entreprise (Cass. soc., 16 mai 1990, n 88-41565; Cass. soc., 3 octobre 1990, nº 88-42334; Cass. soc., 29 novembre 1990, n 88-40618).

116. CC, déc. $\mathrm{n}^{\circ}$ 2015-523 QPC. 
exclus, sachant au surplus que le droit de l'Union ne retient pas une telle distinction ${ }^{117}$. Mais le Conseil va éluder cette question et se focaliser sur la seule différence de traitement existant entre les salariés licenciés pour faute lourde sachant que certains d'entre eux, ceux dont l'employeur est affilié à une caisse de congés, peuvent bénéficier de ladite indemnité ${ }^{118}$. Le Conseil va considérer que la rupture d'égalité entre les salariés licenciés pour faute lourde est constituée et injustifiée ${ }^{119}$, ce qui aura pour effet d'invalider la disposition contestée qui prévoyait que

L'indemnité est due dès lors que la rupture du contrat de travail n'a pas été provoquée par la faute lourde du salarié, que cette rupture résulte du fait du salarié ou du fait de l'employeur ${ }^{120}$.

Par conséquent, même si le Conseil a contrôlé cette disposition par un biais limité, sa censure reste globale, provoquant l'alignement de tous les salariés sur le régime général du bénéfice de l'indemnité compensatrice de congés payés, y compris donc au profit des salariés licenciés pour faute lourde dont l'employeur n'est pas adhérent à une caisse de congés.

Mais force est de constater que le Conseil s'est tout de même bien gardé de se prononcer sur la situation spécifique des salariés licenciés pour faute lourde qui se voient ainsi restreindre l'usage de leurs droits par la loi, comme le droit aux congés payés, alors même que la jouissance de ceux-ci apparaît sans lien avec un quelconque motif de licenciement ${ }^{121}$.

Le droit individuel à la formation fait également partie des acquis dont le législateur a limité le bénéfice pour les salariés licenciés pour faute lourde. Le Conseil a eu l'occasion de se prononcer sur ce dispositif légal dans sa décision $n^{\circ}$ 2016-558/559 QPC du 29 juillet 2016. Le Conseil était saisi des dispositions encadrant la possibilité pour le salarié licencié de demander durant son préavis à recouvrer ses droits à formation non utilisés au cours du contrat de travail, dispositions non applicables aux salariés licenciés pour faute lourde. Plus précisément, l'article L. 6323-17 du Code du travail qui était contesté prévoit qu'
En cas de licenciement non consécutif à une faute lourde, et si le salarié en fait la demande avant la fin du préavis, la somme correspondant au solde du nombre d'heures acquises au titre du droit individuel à la formation et non utilisées, multiplié par le montant forfaitaire visé au deuxième alinéa de l'article L. 6332-14, permet de financer tout ou partie d'une action de bilan de compétences, de validation des acquis de l'expérience ou de formation. À défaut d'une telle demande, la somme n'est pas due par l'employeur.

Les requérants considèrent que ces dispositions constituent une rupture d'égalité dans l'accès à la formation individuelle selon que le salarié est licencié pour faute lourde ou non.

Le Conseil ne va pas se prononcer sur cette rupture d'égalité et va développer un raisonnement assez surprenant en considérant que

L'impossibilité pour le salarié licencié pour faute lourde de demander, postérieurement à l'expiration de son contrat de travail, le bénéfice des heures acquises au titre du droit individuel à la formation et non utilisées à la date d'effet de son licenciement ne résulte pas des dispositions contestées de l'article L. 6323-17 du code du travail.

Il en conclut que «le grief tiré de l'atteinte à l'égal accès à la formation professionnelle est donc inopérant à l'encontre de ces dispositions ${ }^{122}$.

Selon le Conseil, le salarié licencié pour faute lourde est de facto exclu du bénéfice de l'article L. 6323-17 au motif que le droit individuel à la formation y afférant ne peut être déclenché que durant la période de préavis qui n'est pas reconnue à ces salariés. Sur ce seul motif, le Conseil refuse ainsi d'apprécier la situation spécifique des salariés licenciés pour faute lourde et la rupture d'égalité qui en découle. Selon le Conseil, c'est donc cette situation de fait qui est responsable du sort du salarié licencié pour faute lourde et non la législation relative à l'exercice du droit individuel à la formation dont il refuse de contrôler la constitutionnalité. C'est donc l'absence de préavis qui justifie selon le Conseil que les salariés licenciés pour faute lourde soient privés de leur droit individuel à formation. Mais il n'y a pas que les salariés licenciés pour faute lourde

117. CJUE, 26 juin 2001, The Queen c. Secretary of State for Trade and Industry, ex parte Broadcasting, Entertainment, Cinematographic and Theatre Union (BECTU), C-173/99.

118. Les employeurs qui adhérent à une caisse de congés sont ceux qui interviennent dans des secteurs d'activité où les salariés peuvent être confrontés du fait de leur mission à l'intermittence et/ou à la précarité. Il s'agit ainsi des secteurs du bâtiment et des travaux publics; des entreprises de transport; des entreprises de manutention des ports et des dockers et des entreprises de spectacles. Précisons que, dans ces secteurs, tous les salariés ne sont pas nécessairement en situation de précarité et / ou d'intermittence mais, dans la mesure où leur employeur est affilié à une caisse de congés payés, ils pourront tous bénéficier de l'indemnité compensatrice de congés payés même s'ils sont licenciés pour faute lourde (ancien art. L. 3141-28 du Code du travail).

119. Voir CC, déc. $n^{\circ}$ 2015-523 QPC, cons. 9: «Considérant que, la différence de traitement entre les salariés licenciés pour faute lourde selon qu’ils travaillent ou non pour un employeur affilié à une caisse de congés est sans rapport tant avec l'objet de la législation relative aux caisses de congés qu'avec l'objet de la législation relative à la privation de l'indemnité compensatrice de congé payé; que, par suite, les dispositions contestées méconnaissent le principe d'égalité devant la loi; que, sans qu'il soit besoin d'examiner les autres griefs, les dispositions contestées doivent être déclarées contraires à la Constitution».

120. Ancien art. L. 3141-26, al. 2 du Code du travail.

121. Jean Mouly estime d'ailleurs que «si l'on considère que le droit aux congés payés n'est plus, comme jadis, une "récompense" de la fidélité du salarié, mais un véritable droit, octroyé en contrepartie de l'accomplissement d'un travail et lui permettant, entre autres, de préserver sa santé, il faut bien reconnaître que la faute du salarié, quelle qu'en soit la gravité, ne peut pas être de nature à le priver de ce droit. On ne saurait, en effet, transiger avec la santé du travailleur» (J. Mouly, «Invalidation par le Conseil constitutionnel...»).

122. CC, déc. nº 2016-558/559 QPC, \$9 
qui ne bénéficient pas de préavis, c'est également le cas des salariés licenciés pour faute grave. Or, pour ces derniers, bien qu'ils soient dépourvus de préavis comme les salariés licenciés pour faute lourde, le législateur ne semble pas avoir voulu les priver de leur droit à la formation. C'est ce qui ressort de l'interprétation officielle des textes faite en 2011 par le ministère du Travail qui préconise que l'employeur satisfasse à la demande du salarié licencié pour faute grave tendant à bénéficier des heures dues au titre du droit individuel à la formation ${ }^{123}$. Le ministre précise que cette demande doit être effectuée "pendant une période égale à celle du préavis qui aurait été applicable s'il n'avait pas été licencié pour faute grave ${ }^{124}$. L'absence de préavis n'est donc pas en soi un obstacle au bénéfice des droits acquis au titre de la formation individuelle, ce qui vient sérieusement ébranler le raisonnement du Conseil...

Le Conseil a également été saisi en 2016 de plusieurs dispositions législatives relatives à la liberté syndicale et au principe de participation des travailleurs. Dans deux décisions, il a été plus particulièrement amené à se prononcer sur la représentativité ${ }^{125}$ des organisations syndicales de salariés et des organisations professionnelles d'employeurs. Cette question de la représentativité est cruciale, tant pour les syndicats de salariés que pour les syndicats d'employeurs.

S'agissant des organisations syndicales de salariés, seuls

Les syndicats déclarés «représentatifs» peuvent [...] désigner des délégués syndicaux et créer des sections syndicales dans les entreprises, présenter des candidats au premier tour des élections professionnelles, négocier les accords collectifs, participer à la gestion d'organismes de l'État social, siéger dans des organismes de concertation, bénéficier de financements ${ }^{126} \ldots$
Modifiant les règles relatives à la représentativité des syndicats de salariés, la loi du 20 août $2008^{127}$ aurait permis selon certains de passer d'une «logique de représentativité octroyée, descendante et permanente pour instituer une représentativité réglée, ascendante et évolutive ${ }^{128}$. Cette évolution, bien qu'elle ait été critiquée, n'en était pas moins attendue ${ }^{129}$, notamment au regard de «l'accroissement du rôle de la négociation collective ${ }^{130}$. Mettant fin à la présomption irréfragable de représentativité au profit des cinq grandes centrales syndicales, la loi de 2008 a modifié les critères de représentativité et en a introduit de nouveaux. Parmi les sept critères cumulatifs inscrits à l'article L. 2121-1 du Code du travail ${ }^{131}$, on retrouve notamment celui de l'audience qui est mesuré en fonction des résultats obtenus aux élections professionnelles. Il ne s'agit toutefois que du régime prévu pour les salariés du secteur privé. La loi du 5 juillet $2010^{132}$ a modifié le régime de représentativité syndicale dans le secteur public.

Le Conseil a eu l'occasion en 2016 de se prononcer sur ce thème. Plus précisément, dans une décision $\mathrm{n}^{\circ}$ 2016-579 QPC rendue le 5 octobre 2016, il a examiné la conformité d'une disposition législative ${ }^{133}$ interprétée par la Cour de cassation comme «cré[ant] une exception en faveur de la CDC [Caisse des dépôts et consignations] au droit commun de la représentation syndicale $»^{134}$. Au sein de la Caisse des dépôts et consignations et de ses filiales, sont employés des agents publics mais aussi des salariés de droit privé ${ }^{135}$. Si l'article 34 , alinéa 4 de la loi du 28 mai 1996 prévoit des règles propres à la Caisse des dépôts et consignations, l'alinéa suivant traite de l'ensemble du groupe, incluant ainsi les filiales. Selon cet alinéa 5 de l'article 34 ,

La Caisse des dépôts et consignations représentée par son directeur général est [...] habilitée à conclure des accords

123. Réponse du ministère du Travail du $1^{\text {er }}$ février 2011, Journal officiel de l'Assemblée nationale, p. 1068, à la question écrite $\mathrm{n}^{\circ}$ 68695 d'Arlette Grosskost, Journal officiel de l'Assemblée nationale, 12 janvier 2010, p. 258, en ligne: http:/questions.assemblee-nationale.fr/q13/13-68695QE.htm. 124. Ibid.

125. Voir A. Bevort, «De la position commune sur la représentativité au projet de loi: renouveau et continuité du modèle social français», Droit social, 2008, p. 823: «Le régime de la représentativité définit les procédures de reconnaissance des acteurs collectifs déclarés aptes à agir au nom des salariés et des employeurs».

126. Ibid.

127. Loi $\mathrm{n}^{\circ} 2008-789$ du 20 août 2008 portant rénovation de la démocratie sociale et réforme du temps de travail, Journal officiel de la République française, nº 194, 21 août 2008, p. 13064.

128. S. Béroud, J.-P. Le Crom, K. Yon, «Représentativités syndicales, représentativités patronales. Règles juridiques et pratiques sociales. Introduction », Travail et emploi, nº 131, 2012, p. 5-22; cité dans le commentaire de la décision n 2015-519 QPC du 3 février 2016, Mouvement des entreprises de France et autres, p. 3, en ligne: http://www.conseil-constitutionnel.fr/conseil-constitutionnel/root/bank/download/2015519QPC2015519qpc_ccc.pdf.

129. MEDEF, CFDT, CGPME, CFE-CGC, UPA, CFTC, CGT-FO, CGT, «Position commune sur la représentativité, le développement du dialogue social et le financement du syndicalisme», 9 avril 2008.

130. F. Favennec-Héry, «La représentativité syndicale», Droit social, 2009, p. 630.

131. Art. L. 2121-1 du Code du travail: "La représentativité des organisations syndicales est déterminée d'après les critères cumulatifs suivants: $1^{\circ}$ Le respect des valeurs républicaines; $2^{\circ}$ L'indépendance; $3^{\circ}$ La transparence financière; $4^{\circ}$ Une ancienneté minimale de deux ans dans le champ professionnel et géographique couvrant le niveau de négociation. Cette ancienneté s'apprécie à compter de la date de dépôt légal des statuts; $5^{\circ}$ L'audience établie selon les niveaux de négociation conformément aux articles L. 2122-1, L. 2122-5, L. 2122-6 et L. 2122-9; $6^{\circ}$ L'influence, prioritairement caractérisée par l'activité et l'expérience; $7^{\circ}$ Les effectifs d'adhérents et les cotisations».

132. Loi $\mathrm{n}^{\circ}$ 2010-751 du 5 juillet 2010 relative à la rénovation du dialogue social et comportant diverses dispositions relatives à la fonction publique, Journal officiel de la République française, $\mathrm{n}^{\circ}$ 154, 6 juillet 2010, p. 1224.

133. Loi no 96-452 du 28 mai 1996 portant diverses mesures d’ordre sanitaire, social et statutaire, Journal officiel de la République française, $\mathrm{n}^{\circ} 123$, 29 mai 1996, p. 7912, dans sa rédaction résultant de la loi n 2001-420 du 15 mai 2001 relative aux nouvelles régulations économiques, Journal officiel de la République française, $\mathrm{n}^{\circ} 113,16$ mai 2001, p. 7776, art. 34.

134. Commentaire de la décision $n^{\circ}$ 2016-579 QPC du 5 octobre 2016, Caisse des dépôts et consignations, p. 7, en ligne: http://www.conseil-constitutionnel. fr/conseil-constitutionnel/root/bank/download/2016579QPC2016579qpc_ccc.pdf.

135. Ibid., p. 2 
collectifs avec les organisations syndicales représentatives et une ou plusieurs personnes morales liées à elle au sens II de l'article L. 439-1 du code du travail ${ }^{136}$.

Ces accords peuvent notamment porter «sur la désignation et les compétences de délégués syndicaux communs ${ }^{137}$. Sur cette base, un accord collectif a été adopté par la Caisse des dépôts et consignations, instaurant des règles de représentativité dérogatoires à celles prévues par le législateur. Malgré l'adoption d'avenants, cet accord ne prenait pas en compte, au moment du litige, les modifications introduites par le législateur en 2008 en matière de représentativité syndicale ${ }^{138}$. Selon la Cour de cassation, la Caisse des dépôts pouvait bel et bien déroger au droit commun de la représentativité syndicale ${ }^{139}$, règles qui sont d'ordre public ${ }^{140}$. C'est cette interprétation de l'article litigieux qui était contestée par les requérants.

Le Conseil a déjà indiqué à plusieurs reprises ${ }^{141}$ que si le législateur peut autoriser

[...] un accord collectif à déroger à une règle qu'il a lui-même édictée et à laquelle il a entendu conférer un caractère d'ordre public, il doit [toutefois] définir d'une façon précise l'objet et les conditions de cette dérogation ${ }^{142}$.

Or, en l'espèce selon le Conseil,

[...] le législateur n'a pas défini de façon suffisamment précise l'objet et les conditions de la dérogation qu'il a entendu apporter aux règles d'ordre public qu'il avait établies en matière de représentativité syndicale et de négociation collective ${ }^{143}$.

En effet, les accords collectifs prévus par l'article 34 peuvent porter sur «les conditions de désignation des délégués syndicaux communs [...] ce qui inclut, notamment, la définition des critères d'audience et de représentativité» mais aussi «la détermination des compétences de ces délégués syndicaux communs ${ }^{144}$.
Le législateur a donc, selon le Conseil constitutionnel, méconnu l'étendue de sa compétence et le huitième alinéa du préambule de la Constitution de 1946 qui protège le principe de participation des travailleurs. Il abroge alors les dispositions contestées mais, afin d'éviter « de supprimer toute représentation syndicale commune aux agents de droit public et aux salariés de droit privé au sein du groupe de la Caisse des dépôts et consignations ${ }^{145}$, il en reporte les effets au 31 décembre 2017.

Le Conseil s'est également prononcé en 2016 sur la représentativité des organisations professionnelles d'employeurs. Jusqu'en 2014, en l'absence de loi en la matière, c'est le juge administratif qui appliquait aux syndicats d'employeurs les critères prévus pour les syndicats de salariés ${ }^{146}$. Dans un «sursaut tardif ${ }^{147}$, le législateur a adopté un régime juridique spécifique aux organisations d'employeurs $^{148}$. La représentativité des organisations professionnelles d'employeurs, «élément central de leur légitimité ${ }^{149}$, leur permet notamment d'être membres de certaines instances, de bénéficier de financements mais surtout leur confère des prérogatives en matière de négociation collective ${ }^{150}$. L'article L. 2151-1 du Code du travail fixe six critères cumulatifs, « très proches de ceux prévus pour les organisations syndicales de salariés ${ }^{151}$. Toutefois, à la différence des critères prévus pour les syndicats de salariés, l'audience est mesurée en fonction du nombre d'entreprises adhérentes à l'organisation ${ }^{152}$. C'est cette mesure de l'audience par référence au nombre d'entreprises adhérentes qui a fait l'objet de la décision du Conseil rendue le 3 février $2016^{153}$.

Saisi par plusieurs organisations professionnelles d'employeurs dont le Mouvement des entreprises de France (MEDEF), le Conseil a déclaré conformes à la Constitution, et plus particulièrement aux alinéas 6 et 8 du préambule de la Constitution de 1946 et à l'article 6 de la DDHC, plusieurs dispositions du Code du travail.

136. Loi n ${ }^{\circ}$ 96-452 dans sa rédaction résultant de la loi no 2001-420, art. 34, al. 5 .

137. Ibid., art. 34 , al. 6 .

138. Voir le commentaire de la décision no 2016-579 QPC, p. 5-7.

139. Cass. soc., 8 juillet 2015, $\mathrm{n}^{\circ}$ 14-20.837.

140. Cass. soc., 6 janvier 2011, $\mathrm{n}^{\circ} 10-18.205$.

141. Voir par exemple CC, déc. $\mathrm{n}^{\circ} \mathbf{2 0 0 4 - 4 9 4}$ DC du 29 avril 2004, Loi relative à la formation professionnelle tout au long de la vie et au dialogue social.

142. CC, déc. nº 2016-579 QPC, \$7.

143. Ibid., $\$ 9$.

144. Ibid., $\$ 8$.

145. Ibid., $\$ 11$.

146. Commentaire de la décision $\mathrm{n}^{\circ}$ 2015-519 QPC, p. 3

147. L. Dauxerre, "Conformité à la Constitution du critère de mesure de l'audience des organisations professionnelles d'employeurs", La semaine juridique, social, no 8-9, 2016, 1081.

148. Loi n ${ }^{\circ}$ 2014-288 du 5 mars 2014 relative à la formation professionnelle, à l'emploi et à la démocratie sociale, Journal officiel de la République française, $\mathrm{n}^{\circ} 55,6$ mars 2014, p. 4848.

149. M. Grévy, «Syndicats professionnels (III - Prérogatives et action)», Répertoire de droit du travail, octobre 2015; cité dans le commentaire de la décision no 2015-519 QPC, p. 6.

150. Commentaire de la décision $n^{\circ}$ 2015-519 QPC, p. 6-9.

151. Ibid., p. 4.

152. Ce n'est donc pas le même critère qui est prévu pour mesurer l'audience pour les syndicats de salariés et pour les syndicats d'employeurs. Voir B. Teyssié, J.-F. Cesaro, A. Martinon, «La représentativité des organisations professionnelles d'employeurs», La semaine juridique, social, ${ }^{\circ}$ 10, 2011, 1102: "La mesure de l'audience des organisations professionnelles d'employeurs ne peut guère trouver appui sur les résultats des scrutins professionnels auxquels les employeurs sont parfois invités à participer: élections prud'homales, élections organisées dans le cadre des chambres professionnelles [...]. Ces scrutins répondent à des finalités et sont soumis à des modalités qui rendent, pour le moins, extrêmement difficile leur utilisation pour mesurer l'audience des organisations professionnelles d'employeurs».

153. CC, déc. n 2015-519 QPC. 
Cette décision permet notamment de rappeler quel est le champ des bénéficiaires de la liberté syndicale protégée par l'alinéa 6 du préambule de 1946 et de préciser celui du principe de participation prévu à l'alinéa 8 . Selon les requérants, mesurer l'audience des organisations selon le seul critère du nombre des entreprises adhérentes, sans prendre en compte le nombre de salariés ou le chiffre d'affaires des entreprises, méconnait la liberté syndicale. Si la question s'était posée en 1946 de restreindre le bénéfice de la liberté syndicale aux seuls travailleurs ou de l'étendre aux employeurs, la présente décision est l'occasion de rappeler que cette liberté est applicable largement, à « tout homme» ${ }^{154}$. En l'espèce, le Conseil relève d'abord «qu'il était loisible au législateur, pour fixer les conditions de mise en œuvre de la liberté syndicale, de définir des critères de représentativité des organisations professionnelles d'employeurs » ${ }^{155}$. Il n'y a pas, selon le Conseil, de contrariété avec l'alinéa 6 du préambule de 1946. Parmi les motifs du Conseil, le considérant 9 attire l'attention. Le Conseil relève d'abord le but poursuivi par le législateur, plutôt favorable aux petites entreprises:

[...] le législateur a entendu assurer un égal accès à la représentativité des organisations professionnelles d'employeurs quel que soit le nombre des salariés employés par les entreprises adhérentes ou leur chiffre d'affaires.

Il ajoute ensuite qu'

[...] en outre, en vertu du troisième alinéa de l'article L. 226119 du code du travail, le nombre de salariés des entreprises adhérant aux organisations professionnelles d'employeurs est pris en compte en matière de négociation collective $e^{156}$.

C'est cette deuxième partie du considérant qui suscite des interrogations puisque l'on peut se demander pourquoi le Conseil donne cette précision, après avoir rappelé le but poursuivi par le législateur. Comme l'a affirmé Sophie Nadal, on peut, dans un premier temps, avoir l'impression qu'il s'agit d'une «justification complémentaire ${ }^{157}$. Toutefois, ce n'est pas le cas selon elle. En effet, juste après le rappel du contenu du considérant 9 , il est fait mention dans le commentaire de la décision que

Le législateur aurait sans doute pu, sans méconnaître aucune exigence constitutionnelle, pondérer le critère de l'audience en fonction du nombre de salariés des entreprises, mais à l'inverse l'absence de pondération pour apprécier la représentativité n'était pas inconstitutionnelle ${ }^{158}$.

Dès lors, le fait en l'espèce pour le législateur de ne pas prendre en compte dans la détermination de l'audience des organisations professionnelles d'employeurs le nombre de salariés et le chiffre d'affaires n'est pas inconstitutionnel. Toutefois, si le législateur avait souhaité les prendre en compte, la disposition aurait été conforme à la Constitution. C'est d'ailleurs en ce sens que la loi dite Travail du 8 août 2016 a modifié le critère de l'audience, celle-ci pouvant désormais se mesurer

[...] en fonction du nombre d'entreprises volontairement adhérentes ou de leurs salariés soumis au régime français de sécurité sociale et, selon les niveaux de négociation, en application du $3^{\circ}$ des articles L. 2152-1 ou L. 2152-4 ${ }^{159}$.

Le Conseil, chargé d'apprécier la conformité à la Constitution de cette loi, n'a pas été saisi sur ce point mais tout porte à croire que celle-ci aurait été déclarée constitutionnelle, eu égard au «brevet de constitutionnalité » que semble lui conférer la décision commentée du 3 février 2016.

Cette décision permet également au Conseil de préciser l'étendue du champ des bénéficiaires du principe de participation des travailleurs protégé par l'alinéa 8 du préambule de 1946. Pour la première fois, il affirme que celui-ci,

[...] qui consacre un droit aux travailleurs, par l'intermédiaire de leurs délégués, à la participation et à la détermination collectives de leurs conditions de travail, ne confère aucun droit équivalent au bénéfice des employeurs [... $]^{160}$.

$\mathrm{Si}$ «[1]'invocation sans ambages du droit de participation au soutien des droits des organisations d'employeurs était inédite», la solution retenue par le Conseil n'en n'était pas moins "aussi logique que prévisible ${ }^{161}$. Dans son considérant 11, le Conseil rappelle que ce droit

[...] a pour bénéficiaires, sinon la totalité des travailleurs employés à un moment donné dans une entreprise, du moins tous ceux qui sont intégrés de façon étroite et permanente à la communauté de travail qu'elle constitue, même s'ils n'en sont pas les salariés ${ }^{162}$.

S'il ne s'applique pas aux employeurs, ce droit a toutefois un champ étendu. En effet, le Conseil a par exemple retenu dans une décision de 2006 que

[...] s'il était loisible au législateur [...] de ne pas conférer à l'ensemble des travailleurs mis à disposition d'une entreprise la qualité d'électeur pour désigner les délégués du personnel et les représentants des salariés à son comité d'entreprise, il ne pouvait, sans méconnaître le huitième alinéa du Préambule de 1946, limiter le corps électoral aux seuls salariés qui lui sont liés par un contrat de travail ${ }^{163}$.

154. Préambule de la Constitution du 27 octobre 1946, al. 6; voir le commentaire de la décision n 2015-519 QPC, p. 14.

155. CC, déc. $\mathrm{n}^{\circ}$ 2015-519 QPC, cons. 8.

156. Ibid, cons. 9 (nous soulignons).

157. S. Nadal, «La représentativité patronale devant le Conseil constitutionnel», Revue de droit du travail, $\mathrm{n}^{\circ} 5,23$ mai 2016 , p. 354

158. Commentaire de la décision $\mathrm{n}^{\mathrm{o}}$ 2015-519 QPC, p. 15.

159. Art. L. 2151-1 du Code du travail (nous soulignons).

160. CC, déc. nº 2015-519 QPC, cons. 11.

161. S. Nadal, «La représentativité patronale...»

162. CC, déc. nº 2015-519 QPC, cons. 11.

163. CC, déc. $\mathrm{n}^{\circ}$ 2006-545 DC du 28 décembre 2006, Loi pour le développement de la participation et de l'actionnariat salarié et portant diverses dispositions d'ordre économique et social, cons. 30 . 
Il semblerait pourtant que le Conseil ait procédé à un certain «resserrement ${ }^{164}$ du principe de participation protégé par le préambule de 1946, à l'occasion de son examen de la loi Travail.

La loi du 8 août 2016 relative au travail, à la modernisation du dialogue social et à la sécurisation des parcours professionnels prévoit, en son article 64,

[...] la mise en place, dans les réseaux d'exploitants d'au moins trois cents salariés en France, liés par un contrat de franchise, d'une instance de dialogue social commune à l'ensemble du réseau ${ }^{165}$.

Alexandre Fabre ne manque pas de souligner la « singularité» de cette instance de dialogue social, dont la création s'inscrit dans une «logique de transcendance des cadres habituels que forment l'entreprise, l'employeur et le contrat de travail ${ }^{166}$. L'article 64 de cette loi est relatif à la création et à la composition de cette instance mais aussi entre autres aux modalités de prise en charge de son fonctionnement. Selon les requérants, cet article était contraire au principe de participation des travailleurs, à la liberté d'entreprendre du franchiseur et du franchisé, au principe d'égalité ou encore à l'objectif d'intelligibilité. Sur le principe de participation, le Conseil est plutôt « expéditif» lorsqu'il écarte ce grief. Ce principe est limité, comme on l'a déjà précisé, au bénéfice des seuls salariés partageant une même «communauté de travail». Selon les parlementaires requérants, «la mise en place d'une instance de dialogue social n'est possible que si les salariés y participant appartiennent à la même communauté de travail ${ }^{167}$, ce qui ne serait pas le cas des salariés de différents franchisés. Le législateur ne pouvait donc créer selon eux cette instance sans méconnaître le principe de participation et plus précisément le champ de ses bénéficiaires. Le Conseil se contente d'écarter ce grief en relevant que ces dispositions de l'article 64 "n'ont ni pour objet ni pour effet de porter atteinte à l'existence et au fonctionnement des instances représentatives du personnel des franchisés et franchiseurs ${ }^{168}$. On en vient alors à se demander si la création de cette instance de dialogue social prévue par l'article 64 est bien fondée sur le principe de participation. En effet, le Conseil ne répond pas directement au grief des requérants, il se contente d'indiquer que la nouvelle instance de dialogue social ne porte pas atteinte aux instances représentatives déjà existantes, sous-entendant que seules ces dernières relèvent du principe de participation. C'est à l'occasion de l'examen des autres griefs que l'on apprend qu'il n'existe pas entre les salariés de différents franchisés de «communauté de travail ${ }^{169}$. Pourtant, il estime qu'il y a bien entre ces salariés " une communauté d'intérêt des salariés de réseaux de franchise ${ }^{170}$. Or, il semble qu'entre ces deux expressions, la «nuance [soit] subtile, voire illusoire $»^{171}$. En outre, le Conseil ne s'appuie pas sur le principe de participation lorsqu'il rappelle l'objectif poursuivi par le législateur, dans son paragraphe 32 sur la liberté d'entreprendre ${ }^{172}$. En définitive, la décision du Conseil est sur ce point surprenante et peu lisible. On ne comprend guère pourquoi celui-ci a fait l'économie d'affirmer clairement que la disposition litigieuse n'était pas fondée sur l'alinéa 8 du préambule de 1946. C'est sur un autre terrain, et finalement de manière assez marginale, que deux dispositions de l'article 64 ont fait l'objet de réserves d'interprétation ${ }^{173}$ et que l'une a été censurée ${ }^{174}$.

Dans cette même décision, le Conseil a eu à connaître de l'exercice de la liberté syndicale à travers l'article $27 \mathrm{de}$ la loi Travail portant sur la mise à disposition de locaux par les collectivités territoriales et leurs groupements au profit d'organisations syndicales. Cette nouvelle disposition, plus protectrice des syndicats, prévoit, sauf convention contraire, le versement d'une indemnisation par la collectivité lorsque celle-ci retire la mise à disposition des locaux sans proposer de locaux de substitution, et ce, alors que le syndicat les utilisait depuis plus de cinq ans.

164. A. Fabre, «L'instance de dialogue social dans les réseaux de franchise», Constitutions, 2016, p. $446 s q$.

165. CC, déc. $\mathrm{n}^{\circ}$ 2016-736 DC du 4 août 2016, Loi relative au travail, à la modernisation du dialogue social et à la sécurisation des parcours professionnels, $\$ 26$.

166. A. Fabre, "L'instance de dialogue social...».

167. CC, déc. $\mathrm{n}^{\circ} 2016-736 \mathrm{DC}, \$ 27$.

168. Ibid., $\$ 39$.

169. Ibid., $\$ 37$, à propos de la liberté d'entreprendre.

170. Ibid., $\$ 29$, à propos du principe d'égalité.

171. A. Fabre, «L'instance de dialogue social...»: «La nuance est subtile, voire illusoire, et c'est sans doute parce que le Conseil a eu conscience de la proximité des deux expressions, et de leur possible confusion, qu'il les a soigneusement ventilées aux deux extrémités de la décision. Sans vouloir verser dans le procès d'intention, on a le sentiment que le Conseil a voulu masquer une certaine gêne, celle qu'il a sûrement ressentie en reconnaissant qu'il existe une communauté d'intérêt entre les salariés des franchisés... tout en affirmant plus loin que ces derniers ne forment pas une communauté de travail».

172. Ibid.

173. Tout d'abord, le Conseil considère que l'alinéa 2 de l'article 64 qui prévoit l'accord mettant en place cette instance de dialogue social et fixe notamment la composition et le mode de désignation de ses membres, n'est dans son principe «pas contraire à la liberté d'entreprendre sous réserve que les entreprises franchisées participent à la négociation» (CC, déc. $n^{\circ}$ 2016-736 DC, $\left.\$ 34\right)$; l'alinéa 5 prévoit quant à lui qu’à défaut d'accord, les heures de délégation ainsi que leurs modalités d'utilisation seront fixées par un décret en Conseil d'État. Or, selon le Conseil, ces dispositions «ne sauraient être interprétées comme autorisant le pouvoir réglementaire à prévoir [...] des heures de délégation supplémentaires, s'ajoutant à celles déjà prévues pour les représentants des salariés par les dispositions législatives en vigueur» (ibid., $\$ 35)$.

174. En raison de l'absence de communauté de travail entre les salariés de différents franchisés, le Conseil considère que l'alinéa 6 de l'article 64 , qui prévoit qu'à défaut d'accord certaines dépenses telles que les frais de séjour et de déplacement sont à la charge du franchiseur, porte une atteinte disproportionnée à la liberté d'entreprendre (ibid., §37). 
Pour le Conseil, la disposition contestée, «dès lors que le législateur n'avait pas précisé quel était le préjudice indemnisé $»^{175}$, est conforme à la Constitution sous réserve que l'indemnité ne dépasse pas «le préjudice subi à raison des conditions dans lesquelles il est mis fin à l'usage de ces locaux ${ }^{176}$. Les dispositions du paragraphe III de l'article 27 prévoyant l'application rétroactive de la disposition citée précédemment ont été quant à elles déclarées contraires à la Constitution ${ }^{177}$.

Les articles 27 et 64 de la loi Travail, s'ils n'étaient pas les seuls à être contestés par les parlementaires, étaient au cœur de la décision du Conseil et on peut s'étonner, de manière générale, du peu de dispositions dont l'inconstitutionnalité est soulevée. Cette loi, qui a fait l'objet d'une forte mobilisation, ne manquera certainement pas de se retrouver à nouveau devant le Conseil à l'occasion de QPC.

\section{B. Les avancées en demi-teinte en matière de logement et de santé}

Concernant le droit au logement, le Conseil a rendu deux décisions QPC dans lesquelles il rappelle l'impératif d'une protection effective de ce droit. La première décision rendue le 22 janvier 2016 concernait l'article L. 4231-1 du Code du travail qui impose au maître d'ouvrage ou donneur d'ordre de prendre à sa charge l'hébergement des salariés de son cocontractant/ sous-traitant lorsque ces salariés sont logés dans des conditions indignes ${ }^{178}$. Ces dispositions étaient contestées par la Fédération des promoteurs immobiliers qui invoque une rupture d'égalité devant les charges publiques. Le Conseil soulève d'office le principe de responsabilité selon lequel tout fait quelconque de l'homme qui cause à autrui un dommage oblige celui par la faute duquel il est arrivé à le réparer.

Le Conseil va rappeler le principe de sauvegarde de la dignité humaine et s'appuyer sur l'objectif à valeur constitutionnelle consistant à reconnaître à chacun la possibilité de disposer d'un logement décent. Ces deux fondements justifient selon le Conseil qu'il puisse être imposé au maître d'ouvrage ou donneur d'ordre de prendre à sa charge l'hébergement des salariés d'un sous-traitant ou cocontractant jusque-là logés dans des conditions indignes. Même le principe de responsabilité ne peut pas remettre en cause cette obligation de principe. Le Conseil souligne d'ailleurs que la responsabilité du maître d'ouvrage ou donneur d'ordre reste mesurée étant donné que celle-ci
[...] est nécessairement subordonnée au constat par les agents de contrôle compétents d'une infraction aux dispositions de l'article 225-14 du code pénal imputable à l'un de ses cocontractants ou d'une entreprise sous-traitante directe ou indirecte $[\ldots]^{179}$.

Le Conseil rappelle en outre que le maître d'ouvrage ou le donneur d'ordre

[...] peut contester l'engagement de sa responsabilité devant la juridiction compétente; qu'il a la faculté d'agir auprès de son cocontractant ou de l'entreprise sous-traitante, par les moyens contractuels dont il dispose, aux fins de régularisation ${ }^{180}$.

Enfin le Conseil précise que le maître d'ouvrage ou donneur d'ordre n'est pas tenu de prendre en charge l'hébergement collectif des salariés autres que ceux qui sont employés à l'exécution du contrat direct ou de sous-traitance, ni pendant une durée excédant celle de l'exécution dudit contrat. Dans pareilles situations sa responsabilité ne saurait être engagée ${ }^{181}$.

Dans sa décision 2016-581 QPC, le Conseil est également amené à se prononcer sur des conditions de logement difficiles, non plus de salariés mais de personnes étrangères en situation irrégulière ${ }^{182}$. Et, il faut l'avouer, à l'heure où plusieurs responsables politiques viennent prôner la diminution des droits au profit des demandeurs d'asile, voire leur suppression dès qu'il s'agit d'étrangers en situation irrégulière, il est appréciable de voir le Conseil constitutionnel rappeler que la situation irrégulière d'un ressortissant étranger ne lui retire pas sa qualité de personne humaine et ne peut donc pas être l'alibi systématique pour la priver de tout droit. Dans sa décision n ${ }^{\circ}$ 2016-581 QPC le Conseil estime en effet que les étrangers, même en situation irrégulière, peuvent être considérés comme des personnes susceptibles de bénéficier d'une protection de droit commun. Dans cette décision il était question du droit au relogement des personnes dont l'habitat devait faire l'objet d'un aménagement suite à une décision publique. En l'espèce, une société publique locale s'était vue confier par la mairie de Paris le traitement de l'habitat dégradé, insalubre et dangereux. Dans le cadre de cette mission, la société était amenée à s'intéresser au sort d'un hôtel occupé par des personnes étrangères en situation irrégulière. Pour procéder aux aménagements nécessaires, la société souhaitait expulser ces personnes. Le Conseil devait se prononcer sur la possibilité de déroger à l'obligation de relogement lorsque sont concernées des personnes dépourvues de titre de séjour. Le Conseil va refuser d'opérer une distinction avec les personnes en situation régulière et il va estimer qu'

175. Commentaire de la décision no 2016-736 DC, p. 11, en ligne: http://www.conseil-constitutionnel.fr/conseil-constitutionnel/root/bank/ download/2016736DC2016736dc_ccc.pdf.

176. CC, déc. $\mathrm{n}^{\circ}$ 2016-736 DC, $\$ 17$.

177. Ibid., $\$ 22$ à 24 .

178. CC, déc. $\mathrm{n}^{\circ}$ 2015-517 QPC du 22 janvier 2016, Fédération des promoteurs immobiliers: Droit social, 2016, p. 372-375, comm. F. Muller; S. Schiller, La semaine juridique, édition générale, $\mathrm{n}^{\circ} 5,2016,113$.

179. CC, déc. n 2015-517 QPC, cons. 11.

180. Ibid., cons. 12 .

181. Ibid., cons. 14

182. CC, déc. n 2016-581 QPC du 5 octobre 2016, Société SOREQA SPLA. 
[...] en adoptant les dispositions contestées, le législateur a entendu protéger les occupants évincés et compenser la perte définitive de leur habitation du fait de l'action de la puissance publique. Ainsi, l'obligation de relogement, en cas d'éviction définitive, met en œuvre l'objectif de valeur constitutionnelle que constitue la possibilité pour toute personne de disposer d'un logement décent ${ }^{183}$.

Par ailleurs, le Conseil va rejeter le recours à des considérations pratiques pour admettre qu'il puisse être dérogé à l'obligation de relogement. Il ne va pas davantage juger recevable l'argument des requérants selon lequel le délit de solidarité viendrait se heurter à cette obligation de relogement. Le Conseil rappelle en effet que

[...] le fait de reloger dans le cadre et les conditions déterminées par l'article L. 314-2 du code de l'urbanisme ne peut caractériser une infraction pénale. L'obligation de relogement prévue par les dispositions contestées ne peut donc exposer à des poursuites pénales pour délit d'aide au séjour irrégulier ${ }^{184}$.

Après de longs mois d'âpres discussions parlementaires et de grève de professionnels de santé, le 17 décembre 2015, le Parlement a définitivement adopté le projet de loi de modernisation de notre système de santé. Saisi le 21 décembre 2015 par 141 sénateurs et 172 députés, le Conseil constitutionnel s'est prononcé sur cette loi par la décision $\mathrm{n}^{\circ}$ 2015-727 DC du 21 janvier 2016. Alors qu'il déclare de nombreuses dispositions conformes à la Constitution, il en censure certaines.

Au titre des dispositions déclarées constitutionnelles, on relève celles relatives aux mesures de lutte contre le tabagisme. Pour valider celles portant sur les modalités d'entrée en vigueur de l'interdiction des arômes et des additifs dans les produits du tabac, le Conseil a procédé à une application classique de sa jurisprudence relative aux lois de transposition de directive. Précisément, la règle d'entrée en vigueur retenue par le législateur n'est pas manifestement incompatible avec les dispositions inconditionnelles et précises de la directive que la loi avait pour objet de transposer. Le Conseil déclare également conforme à la Constitution la disposition supprimant des dérogations à l'interdiction de publicité pour le tabac dans les débits de tabac dans la mesure où elle poursuit l'objectif de protection de la santé et ne porte pas d'atteinte manifestement disproportionnée à la liberté d'entreprendre. Il fait ici une application également classique de sa jurisprudence sur la liberté d'entreprendre selon laquelle il n'opère qu'un contrôle restreint lorsque la limitation apportée par le législateur à cette liberté est liée à une exigence constitutionnelle. Enfin, pour ce qui concerne le paquet neutre, le Conseil ne relève pas d'incompétence négative de la part du législateur, ni de privation de propriété au sens de l'article 17 de la DDHC ${ }^{185}$ ou encore d'atteinte manifestement disproportionnée au droit de propriété au sens de l'article 2 de la $\mathrm{DDHC}^{186}$. L'exigence constitutionnelle de protection de la santé publique permet là encore de considérer qu'il n'est pas porté une atteinte manifestement disproportionnée à la liberté d'entreprendre.

Sont également déclarées conformes à la Constitution les dispositions instituant les « salles de consommation à moindre risque ${ }^{187}$. Étaient contestées les dispositions qui prévoient des immunités pénales. Celles-ci concernent les consommateurs, qui ne peuvent être poursuivis pour usage illicite et détention illicite de stupéfiants, et les professionnels qui interviennent dans ces salles, et qui ne peuvent être poursuivis pour complicité d'usage illicite de stupéfiants et pour facilitation de l'usage illicite de stupéfiants. Selon les requérants, ces immunités méconnaissent le principe d'égalité devant la loi pénale dans la mesure où il n'existe aucune différence de situation ni de motif d'intérêt général qui pourraient justifier ces dérogations. Ce n'est pas la première fois que le Conseil se prononce sur des immunités pénales et il reprend dans le considérant 25 , son considérant de principe en la matière:

Considérant, en premier lieu, qu'en application de l'article 34 de la Constitution, il revient au législateur de fixer, dans le respect des principes constitutionnels, les règles concernant la détermination des crimes et délits qu'il crée, ainsi que les peines qui leur sont applicables; qu'il peut aussi prévoir, sous réserve du respect des règles et principes de valeur constitutionnelle et, en particulier, du principe d'égalité, que certaines personnes physiques ou morales bénéficieront d'une immunité pénale; qu'il résulte de l'article 34 de la Constitution, ainsi que du principe de la3 légalité des délits et des peines posé par l'article 8 de la Déclaration de 1789 , la nécessité pour le législateur de fixer lui-même le champ d'application de la loi pénale, de définir les crimes et délits en termes suffisamment clairs et précis pour permettre la détermination des auteurs d'infractions et exclure l'arbitraire dans le prononcé des peines, et de fixer dans les mêmes conditions le champ d'application des immunités qu'il instaure.

Le Conseil rappelle par ailleurs au considérant 35 que normalement le caractère expérimental d'une disposition peut permettre de mettre de côté le grief tiré de l'atteinte au principe d'égalité, y compris en matière pénale. Le juge procède pourtant ici au contrôle des immunités contestées au regard du principe d'égalité devant la loi dans la mesure où l'objectif du législateur est de pérenniser la différence de traitement instituée. Pour autant, cela n'a pas fait obstacle à la déclaration de constitutionnalité de la disposition.

Par ailleurs, le Conseil constitutionnel valide la suppression du délai de réflexion préalable d'une semaine entre la demande d'interruption volontaire de grossesse et la confirmation écrite. Conformément à sa jurisprudence 
antérieure $^{188}$, il décide que l'équilibre entre la sauvegarde de la dignité de la personne humaine et la liberté de la femme qui découle de l'article 2 de la DDHC n'est pas rompu ${ }^{189}$ dès lors qu'il est assuré ici que la demande d'interruption de grossesse et la confirmation écrite n'interviennent pas au cours de la même consultation. Cette considération permet d'assurer le respect d'un délai de réflexion minimum.

Le Conseil constitutionnel déclare également conforme à la Constitution la disposition de l'article L. 6112-2 du Code de la santé publique selon laquelle

I. - Les établissements de santé assurant le service public hospitalier et les professionnels de santé qui exercent en leur sein garantissent à toute personne qui recourt à leurs services: [...] $4^{\circ}$ L'absence de facturation de dépassements des tarifs fixés par l'autorité administrative et des tarifs des honoraires prévus au $1^{\circ}$ du I de l'article L. 162-14-1 du code de la sécurité sociale. Le patient bénéficie de ces garanties y compris lorsqu'il est transféré temporairement dans un autre établissement de santé ou dans une autre structure pour des actes médicaux.

\section{Il apparaît que}

L'atteinte au principe d'égalité, à supposer qu'elle existe, ne pouvait résulter que de la disposition selon laquelle les praticiens statutaires exerçant dans les établissements publics de santé sont autorisés à avoir une activité libérale. Dans son contrôle exercé au regard du principe d'égalité, le Conseil constitutionnel ne vérifie pas si d'autres dispositions que celles dont il est saisi sont susceptibles de créer, de manière autonome, une discrimination ${ }^{190}$.

Le juge constitutionnel valide également la disposition procédant à l'extension des missions de contrôle de la Cour des comptes et des chambres régionales des comptes aux établissements sanitaires, sociaux ou médico-sociaux privés. Il rejette le grief d'incompétence négative en précisant, par un considérant de principe, que si

[...] la nécessité pour l'État de contrôler l'emploi des ressources que ces personnes morales de droit privé perçoivent est de nature à justifier l'instauration d'un régime spécifique de contrôle [...] il appartiendra toutefois au pouvoir réglementaire de veiller, en fixant les modalités de mise en œuvre de ces contrôles, au respect des principes constitutionnels de la liberté d'entreprendre ou de la liberté d'association des personnes morales de droit privé concernées $[\ldots]^{191}$.

En ce qui concerne les recherches biomédicales sur les gamètes et les embryons, et après avoir écarté le grief portant sur un «cavalier», le Conseil constitutionnel, conformément à ce qu'il avait déjà apprécié dans sa décision no 2013-674 DC, considère qu'il n'y a aucune méconnaissance du principe de dignité de la personne humaine en raison de l'existence d'un certain nombre de garanties en ce sens ${ }^{192}$.

Enfin, la disposition relative au renforcement de la transparence des liens d'intérêt entre les laboratoires pharmaceutiques et les autres acteurs du monde de la santé n'est pas jugée attentatoire au droit au respect de la vie privée. Précisément, le Conseil considère qu'

[...] eu égard aux exigences particulières qui pèsent sur les acteurs du secteur de la santé et à la gravité des conséquences des conflits d'intérêt dans ce secteur, le législateur a opéré une conciliation qui n'est pas manifestement déséquilibrée entre les principes constitutionnels en cause $[\ldots]^{193}$.

C'est la spécificité du domaine de la santé qui justifie la solution retenue par le Conseil constitutionnel [... $]^{194}$.

En revanche, est déclarée inconstitutionnelle la disposition rendant obligatoire le dispositif du tiers payant pour les organismes d'assurance maladie complémentaire à compter du $1^{\text {er }}$ janvier 2017. Le «tiers payant» est le dispositif qui permet de dispenser d'avance de frais les bénéficiaires de l'assurance maladie recevant des soins de ville, à hauteur des montants remboursés par l'assurance maladie et les organismes complémentaires. Les requérants ont soulevé l'incompétence négative du législateur, en ce que ce dernier n'aurait pas suffisamment encadré certains aspects du dispositif pour les organismes d'assurance maladie complémentaire. Le Conseil constitutionnel a jugé que si

[...] les dispositions contestées précisent les conditions dans lesquelles est garanti au professionnel de santé le paiement de la part des honoraires prise en charge par les régimes obligatoires de base d'assurance maladie $[\ldots]^{195}$

en revanche,

[...] ni les dispositions contestées ni aucune autre disposition ne prévoient des mesures équivalentes en ce qui concerne l'application du tiers payant aux dépenses prises en charge par les organismes d'assurance maladie complémentaire $[\ldots]^{196}$.

Il procède en conséquence à la censure partielle de l'article 83 de la loi.

Quant à la disposition habilitant le gouvernement à prendre par ordonnances des mesures permettant de définir des règles budgétaires et comptables qui régissent les relations entre établissements publics parties à un même groupement hospitalier de territoire, elle est de même déclarée inconstitutionnelle. Constatant que

188. CC, déc. $\mathrm{n}^{\circ}$ 2001-446 DC du 27 juin 2001, Loi relative à l'interruption volontaire de grossesse et à la contraception.

189. CC, déc. $\mathrm{n}^{\circ}$ 2015-727 DC, cons. 43.

190. Commentaire de la décision $n^{\circ}$ 2015-727 DC du 21 janvier 2016, Loi relative à la modernisation de notre système de santé, p. 26, en ligne: http:// www.conseil-constitutionnel.fr/conseil-constitutionnel/root/bank/download/2015727DC2015727dc_ccc.pdf.

191. CC, déc. $\mathrm{n}^{\circ}$ 2015-727 DC, cons. 70.

192. Ibid., cons. 85 .

193. Ibid., cons. 92.

194. Commentaire de la décision $n^{\circ}$ 2015-727 DC, p. 30.

195. CC, déc. $\mathrm{n}^{\circ}$ 2015-727 DC, cons. 48.

196. Ibid. 
[...] ni le paragraphe IX de l'article 107 ni aucune autre disposition de la loi déférée ne fixe la date à laquelle le projet de loi de ratification des ordonnances que le Gouvernement est habilité à prendre devra être déposé devant le Parlement $[\ldots]^{197}$

le Conseil considère en effet que «cette habilitation méconnaît les exigences qui résultent de l'article 38 de la Constitution $»^{198}$.
Il a en outre, après s'en être saisi d'office, déclaré contraires à la Constitution, pour avoir été introduits en nouvelle lecture par l'Assemblée nationale en méconnaissance de la règle dite de l' «entonnoir », le II de l'article 46 (rapport au Parlement relatif à la contribution du transport aérien à la pollution atmosphérique et à ses effets sur la santé humaine) et le II de l'article 59 (rapport au Parlement relatif aux effets sur la santé du bisphénol A non chauffé). 